\title{
Analysis of a Stabilized Finite Element Approximation of the Transient Convection-Diffusion Equation using an Ale Framework
}

S. Badia R. Codina 


\section{Analysis of a Stabilized Finite Element Approximation of the Transient Convection-Diffusion Equation using an Ale Framework}

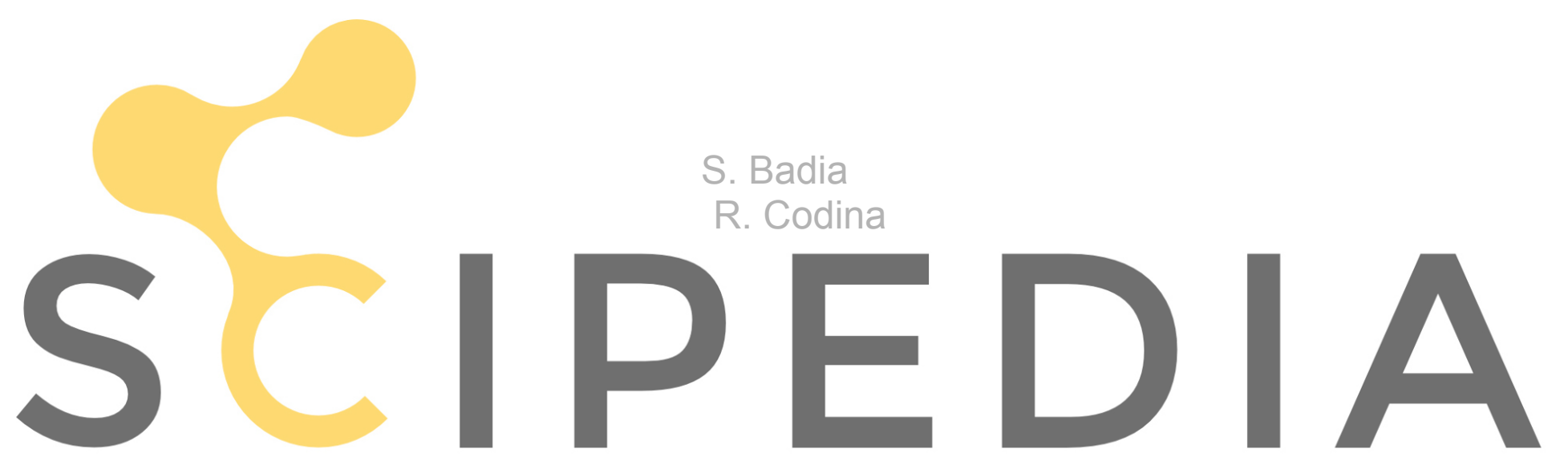

Register for free at https//www.scipedia.com to download the version without the watermark

Publication CIMNE №-285, May 2006 


\title{
ANALYSIS OF A STABILIZED FINITE ELEMENT APPROXIMATION OF THE TRANSIENT CONVECTION-DIFFUSION EQUATION USING AN ALE FRAMEWORK *
}

\author{
SANTIAGO BADIA ${ }^{\dagger}$ AND RAMON CODINA $\ddagger$
}

\begin{abstract}
In this paper we analyze a stabilized finite element method to approximate the convection-diffusion equation on moving domains using an ALE framework. As basic numerical strategy, we discretize the equation in time using first and second order backward differencing (BDF) schemes, whereas space is discretized using a stabilized finite element method (the orthogonal subgrid scale formulation) to deal with convection dominated flows. The semi-discrete problem (continuous in space) is first analyzed. In this situation it is easy to identify the error introduced by the ALE approach. After that, the fully discrete method is considered. We obtain optimal error estimates in both space and time in a mesh dependent norm. The analysis reveals that the ALE approach introduces an upper bound for the time step size for the results to hold. The results obtained for the fully discretized second order scheme (in time) are associated to a weaker norm than the one used for the first order method. Nevertheless, optimal convergence results have been proved. For fixed domains, we recover stability and convergence results with the strong norm for the second order scheme, stressing the aspects that make the analysis of this method much more involved
\end{abstract}

Key words. Stabilized finite elements, second order BDF, ALE

1. Introduction. In this paper we propose and analyze two time integration schemes, of first and of second order, for the numerical approximation of the transient convectiondiffusion equation in moving domains. This equation is written in an ALE framework, in which the temporal derivatives are expressed with respect to the reference of a moving domain $\Omega_{t}$ obtained from a mapping of the domain at the initial time. The space discretization is carried out using a stabilize dominated flows.

The ALE framework, in come widely popular when can find a lot of numerical ex on the dark side for a long time. For instance, the meaning and effect of the Geometric con-

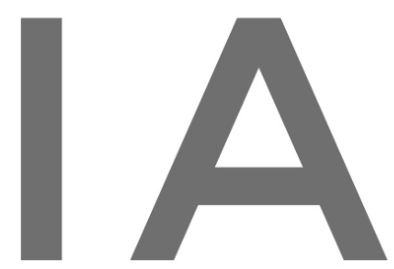
servation Law (GCL) or how the accuracy of a numerical method in fixed domains is spoiled

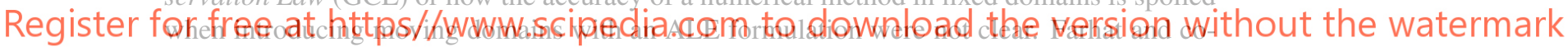
authors have shown in [13] that the GCL makes the numerical scheme preserve a maximum principle. In [16], the authors have shown that this condition is not necessary to obtain second order ALE schemes in a finite volume framework. More recently, in a finite element setting and taking the transient convection-diffusion equation as the model equation, works like [14] and [23] have also allowed to clarify the effect of the GCL on the stability properties, the different behavior between conservative and non-conservative forms, and also some convergence results have been proved. Further analyses, for second order schemes, have been developed in later works, as [15] and [2]. Herein we use the mathematical setting used, e.g., in [23] for the description of this method.

The ALE framework does not introduce any error by itself at the continuous level. However, when the problem is discretized in time, some errors due to the ALE description arise. At this step, for fixed domains, the only source error is the time derivative of the unknown. In addition, for moving domains, also the error from the evaluation of the mesh velocity has

*Universitat Politècnica de Catalunya, International Center for Numerical Methods in Engineering (CIMNE), Jordi Girona 1-3, Edifici C1, 08034 Barcelona, Spain.

$\dagger$ sbadia@cimne.upc.edu

$\ddagger$ ramon.codina@upc.edu 
to be accounted for. This velocity is calculated as the time derivative of the space position of a particle. Thus, an error is induced when this time derivative is calculated numerically.

On the other hand, in practical applications the mesh velocity belongs to the finite element space and does not introduce any interpolation error. Thus, we consider that the ALE formulation is better understood analyzing the problem semi-discretized in time. However, most numerical analysis (see [14], [15] and [2]) first study the semi-discrete problem in space, and then the fully discretized problem.

The convection-diffusion equation (as the Navier-Stokes equations) when discretized in space with the standard Galerkin formulation shows numerical oscillations if the convective term is dominant. With the aim of developing a finite element method free of spurious oscillations many methods have been proposed during the last twenty years, such as SUPG (see [5]), Galerkin/Least-squares (see [22]) or the Subgrid Scale stabilization (see [20]). A comparative of different stabilization methods can be found in [6]. The Orthogonal Subgrid Scale method (OSS) used in this paper belongs to this last family and was introduced by Codina in [7]. The method is designed taking as starting point the Subgrid Scale variational setting proposed by Hughes in [21] and modeling the subgrid problem in a particular way, in particular taking the subgrid scales orthogonal to the finite element space. The common aspect of all these methods is found in the convergence analysis of the discrete problem in space. For the Galerkin approximation, the error estimate bound depends on the physical properties (the Péclet number for the convection-diffusion equation), and increases as the convective term is more dominant. In fact, the stability bound blows up as diffusion goes to zero, reflecting the fact that the continuous problem is a singularly perturbed one. But when using stabilized methods this negative feature does not appear anymore. This is explained because the new terms introduced by the stabilization control the convective term norm. In the present analysis we have been able to convective term, which is

As far as we know, most

problems using the framew

ing notably the computer cost
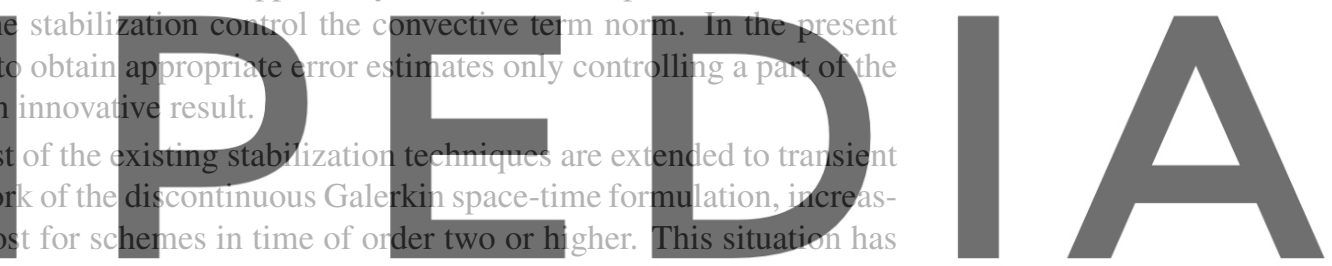

been improved by Guermond in [18], where he analyzes the introduction of a certain numeri-

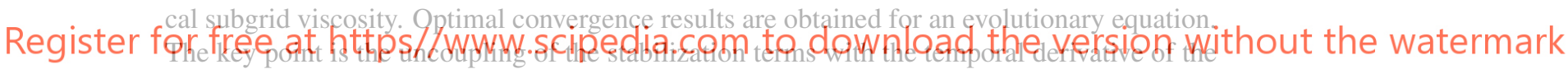
unknown. Another stabilization method with this feature is presented in [3].

Codina and Blasco analyze in [11] the transient convection-diffusion-reaction equation discretized in space using the OSS method and in time with the backward Euler time integration. Further, they consider the tracking of the subscales in time. Optimal convergence and stability results are obtained.

The present paper can be viewed as an extension of [11]. We generalize the situation to moving domains (using an ALE approach). In addition, first and second order backward differencing (BDF) time integration schemes are considered, which will be denoted by BDF1 and BDF2, respectively. The blend of a stabilized finite element method with the use of an ALE framework is one of the innovative aspects of this paper.

In order to analyze the stabilized method for transient problems, the following strategy is adopted in [11]: first the semi-discrete problem is studied (where no stabilization terms appear) and later the fully discrete method is analyzed. As it is shown in [11], this provides a natural way to deal with the subscales whose approximation enhances the stability and accuracy of the formulation. The main drawback of this strategy is that space regularity for the convergence analysis needs to be assumed for the semidiscrete solution, not for the continuous one. 
The first time integration scheme considered uses the classical backward Euler formula for the approximation of both the time derivative of the unknown and the calculation of the mesh velocity. We label this method as:

- BDF1-BDF1 $1_{\delta t}$ for the problem semi-discretized in time,

- $\mathrm{BDF} 1-\mathrm{BDF} 1_{\delta t, h}$ for the fully discretized problem using the classical Galerkin approximation in space,

- BDF1-BDF1-OSS $\delta t, h$ for the fully discretized problem using the OSS method in space,

- BDF1-OSS ${ }_{\delta t, h}$ for the fully discretized problem using the OSS method in space on fixed domains (not in an ALE framework).

In the second method the time integration makes use of the second order BDF formula. Again, we use the following notation:

- $\mathrm{BDF} 2-\mathrm{BDF} 2_{\delta t}$ for the problem semi-discretized in time,

- BDF2-BDF2 $\delta t, h$ for the fully discretized problem using the classical Galerkin approximation in space,

- BDF2-BDF2-OSS $\delta t, h$ for the fully discretized problem using the OSS method in space,

- BDF2-OSS $\delta t, h$ for the fully discretized problem using the OSS method in space on fixed domains (not in an ALE framework).

Let us underline what is new in each case. The BDF1-BDF1 $\delta t, h$ method has been analyzed in [14]. As explained above, we change the order of the discretization: first we analyze BDF1-BDF1 $1_{\delta t}$, and then BDF1-BDF1-OSS ${ }_{\delta t, h}$, introducing the appropriate stabilization terms. For fixed domains, the BDF1-OSS $\delta t, h$ has been analyzed in [11]. However, the analy-

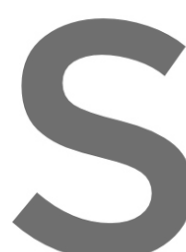
sis herein is slightly different method BDF2-BDF2 $\delta t$ is $\mathrm{OSS}_{\delta t, h}$. We specially remar properties can be obtained $w$ mains, the stability and conving time behavior has been considered
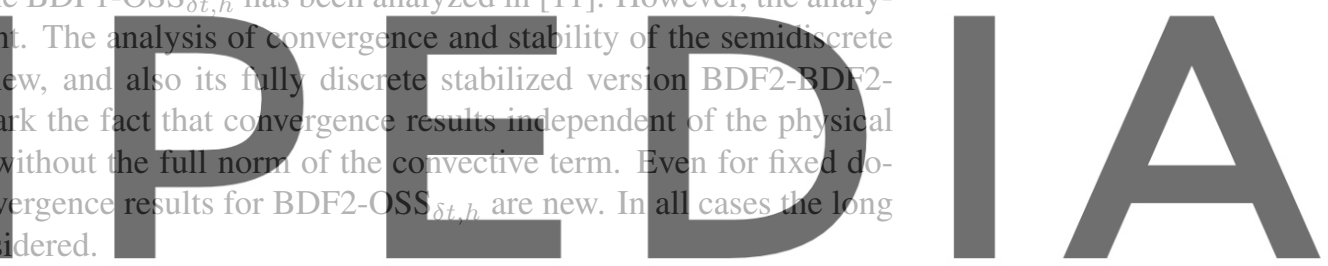

Numerical experimentation with the ALE methods (for diffusion dominated problems us[23], showing the expected behavior. The application of BDF1-OSS $\delta t, h$ and BDF2-OSS $\delta t, h$, can be found in [8] and [10] for the solution of fluid problems. Finally, the blend of these methods, BDF1-BDF1-OSS $\delta t, h$ and BDF2-BDF2-OSS $\delta t, h$, has been used for simulating engineering problems in [1], with excellent results.

The paper is organized as follows. In $\S 2$ we state the governing equations for moving domains in an ALE framework. Some important ingredients in order to define the ALE approach are introduced. The semi-discrete problem is formulated both for BDF1 and BDF2. The section ends with the presentation of the OSS stabilization method and the fully discrete problem. $\S 3$ is devoted to the semi-discrete problem. First and second order methods are considered for which stability and optimal convergence estimates are obtained. $\S 4$ presents an analogous analysis to that of $\S 3$ but for the fully discrete problem. Finally, some conclusions are drawn in $\S 5$.

\section{Problem statement.}

2.1. The continuous problem. In order to study the ALE framework together with a stabilized finite element method, we take as a model test problem the transient convectiondiffusion equation. The problem written in an Eulerian framework consists in finding a func- 
tion $u$ such that

$$
\begin{aligned}
\frac{\partial u}{\partial t}-\nu \Delta u+\boldsymbol{a} \cdot \nabla u & =f & & \text { in } \Omega_{t} \times(0, T), \\
u & =0 & & \text { on } \partial \Omega_{t} \times(0, T), \\
u\left(\boldsymbol{x}_{0}, 0\right) & =u_{0} & & \text { in } \Omega_{0} \times\{0\},
\end{aligned}
$$

where $\Omega_{t} \subset \mathbb{R}^{d}$ (d=2,3) is a bounded and polyhedral domain (moving in time), $[0, T]$ is the time interval of analysis, $\boldsymbol{a}$ is a divergence-free velocity field and $\nu>0$ is the diffusion coefficient. Homogeneous boundary conditions are assumed to clarify the analysis. We also assume the following regularity of the data:

$$
f \in L^{2}\left(0, T ; H^{-1}\left(\Omega_{t}\right)\right), \quad u_{0} \in L^{2}\left(\Omega_{0}\right), \quad \boldsymbol{a} \in \boldsymbol{L}^{\infty}\left(\Omega_{t}\right),
$$

assuring the existence of a unique solution $u(t) \in L^{2}\left(0, T ; H^{1}\left(\Omega_{t}\right)\right) \cap \mathcal{C}^{0}\left(0, T ; L^{2}\left(\Omega_{t}\right)\right)$.

We introduce some key ingredients of an ALE framework. Let $\mathcal{A}_{t}$ be a family of mappings, which for all $t \in[0, T]$ map a point $x_{0} \in \Omega_{0}$ into a point $x \in \Omega_{t}$,

$$
\mathcal{A}_{t}: \Omega_{0} \longrightarrow \Omega_{t}, \quad x\left(x_{0}, t\right)=\mathcal{A}_{t}\left(x_{0}\right) .
$$

We assume that $\mathcal{A}_{t}$ is invertible with inverse $\mathcal{A}_{t}^{-1}$. For $t_{1}, t_{2} \in[0, T]$ we define

$$
\mathcal{A}_{t_{1}, t_{2}}: \Omega_{t_{1}} \longrightarrow \Omega_{t_{2}}, \quad \mathcal{A}_{t_{1}, t_{2}}=\mathcal{A}_{t_{2}} \circ \mathcal{A}_{t_{1}}^{-1} \text {. }
$$

We note that the family of mappings is arbitrary. Several techniques have been suggested in order to construct this AIE mappin particles, the resulting formulation

Let us consider a function $f$ corresponding function in th
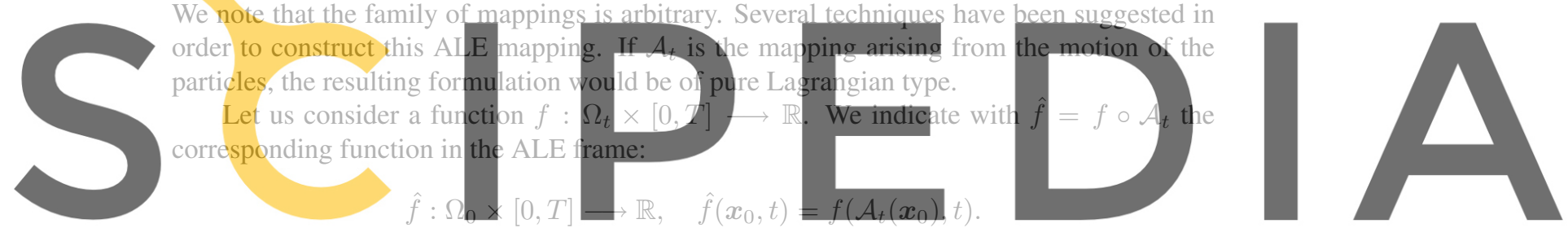

Register for free at https//WWW.scipedia.com to download the version without the watermark

$$
\left.\frac{\partial f}{\partial t}\right|_{\boldsymbol{x}_{0}}: \Omega_{t} \times[0, T] \longrightarrow \mathbb{R},\left.\quad \frac{\partial f}{\partial t}\right|_{\boldsymbol{x}_{0}}(x, t)=\frac{\partial \hat{f}}{\partial t}\left(x_{0}, t\right) .
$$

The domain velocity $\boldsymbol{w}$ is calculated using the following expression:

$$
\boldsymbol{w}(\boldsymbol{x}, t)=\left.\frac{\partial \boldsymbol{x}}{\partial t}\right|_{\boldsymbol{x}_{0}}=\frac{\partial \mathcal{A}_{t}\left(\boldsymbol{x}_{0}\right)}{\partial t},
$$

and the Jacobian of the ALE mapping is given by

$$
J_{t}=\operatorname{det}\left(\boldsymbol{J}_{t}\right), \quad \boldsymbol{J}_{t}=\frac{\partial \boldsymbol{x}}{\partial \boldsymbol{x}_{0}} .
$$

We recall the Reynolds transport formula. Let $\psi(\boldsymbol{x}, t)$ be a function defined in $\Omega_{t}$. Then, for any subdomain $V_{t} \subseteq \Omega_{t}$ such that $V_{t}=\mathcal{A}_{t}\left(V_{0}\right)$ with $V_{0} \subseteq \Omega_{0}$ it holds that

$$
\frac{d}{d t} \int_{V_{t}} \psi(\boldsymbol{x}, t) \mathrm{d} V=\int_{V_{t}}\left(\left.\frac{\partial \psi}{\partial t}\right|_{\boldsymbol{x}_{0}}+\psi \nabla \cdot \boldsymbol{w}\right) \mathrm{d} V .
$$


In particular, if $v: \Omega_{t} \longrightarrow \mathbb{R}$, that is, if $v$ does not depend explicitly on time, we have that

$$
\frac{d}{d t} \int_{\Omega_{t}} v \mathrm{~d} \Omega=\int_{\Omega_{t}} v \nabla \cdot \boldsymbol{w} \mathrm{d} \Omega
$$

With all this notation introduced, we are ready to write (2.1) in the ALE framework. It now reads

$$
\begin{aligned}
\left.\frac{\partial u}{\partial t}\right|_{\boldsymbol{x}_{0}}-\nu \Delta u+(\boldsymbol{a}-\boldsymbol{w}) \cdot \nabla u & =\boldsymbol{f} & & \text { in } \Omega_{t} \times(0, T), \\
u & =0 & & \text { on } \partial \Omega_{t} \times(0, T), \\
\boldsymbol{u}\left(\boldsymbol{x}_{0}, 0\right) & =\boldsymbol{u}_{0} & & \text { in } \Omega_{0} \times\{0\} .
\end{aligned}
$$

The funcional space

$$
\mathcal{V}\left(\Omega_{t}\right):=\left\{v: \Omega_{t} \rightarrow \mathbb{R}, v=\hat{v} \circ \mathcal{A}_{t}^{-1}, \hat{v} \in H_{0}^{1}\left(\Omega_{0}\right)\right\}, \quad t \in(0, T)
$$

allows us to write (2.3) in its variational form. The variational problem reads: find $u(t) \in$ $\mathcal{V}\left(\Omega_{t}\right)$ for all $t \in(0, T)$ such that

$$
\left(\frac{\partial u(t)}{\partial t}, v\right)_{\Omega_{t}}+\nu(\nabla u(t), \nabla v)_{\Omega_{t}}+((a-w(t)) \cdot \nabla u(t), v)_{\Omega_{t}}=\langle f(t), v\rangle_{\Omega_{t}},
$$

$\forall v \in \mathcal{V}\left(\Omega_{t}\right)$, where $(\cdot, \cdot) \Omega_{t}$ stands for the $L^{2}\left(\Omega_{t}\right)$ inner product and $\langle\cdot, \cdot\rangle_{\Omega_{t}}$ for the duality pairing in $H^{-1}\left(\Omega_{t}\right) \times H_{0}^{1}\left(\Omega_{t}\right)$.

Let us re-scale the time coefficient $1 / T$ has to be is to display which terms in t the long time behavior. After re-scaling, problem (2.4) is th
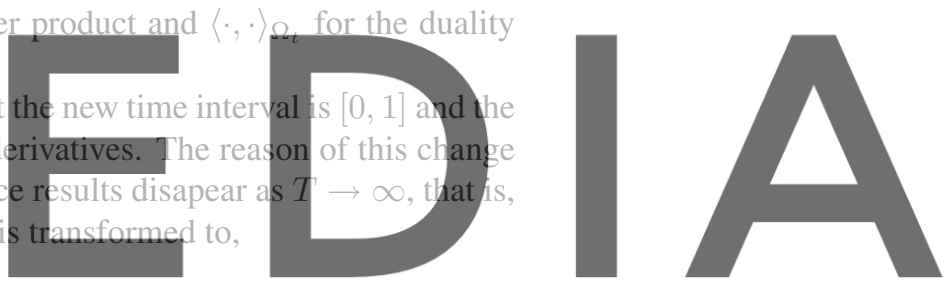

Register for free ${ }^{\partial t}$ th https//www.scipedia.com to download the version without the watermark and now the domain velocity is,

$$
\boldsymbol{w}(\boldsymbol{x}, t)=\left.\frac{1}{T} \frac{\partial \boldsymbol{x}}{\partial t}\right|_{\boldsymbol{x}_{0}}
$$

We take into account this re-scaling in property (2.2), which now reads

$$
\frac{1}{T} \frac{d}{d t} \int_{\Omega_{t}} v \mathrm{~d} \Omega=\int_{\Omega_{t}} v \nabla \cdot \boldsymbol{w} \mathrm{d} \Omega
$$

2.2. The semi-discrete problem in time. Let us introduce some notation that we will used throughout the work. Consider a uniform partition of $[0,1]$ into $N$ time intervals of length $\delta t$. Let us denote by $f^{n}$ the approximation of a time dependent function $f$ at time level $t^{n}=n \delta t$. We will also denote

$$
\begin{aligned}
\delta f^{n+1} & \equiv \delta^{(1)} f^{n+1}=f^{n+1}-f^{n}, \\
\delta^{(i+1)} f^{n+1} & =\delta^{(i)} f^{n+1}-\delta^{(i)} f^{n}, i=1,2,3, \ldots
\end{aligned}
$$


The discrete operators $\delta^{(i+1)}$ are centered. We will also use the backward difference operators

$$
\begin{aligned}
D_{1} f^{n+1} & =\frac{\delta f^{n+1}}{\delta t}=\frac{f^{n+1}-f^{n}}{\delta t}, \\
D_{2} f^{n+1} & =\frac{3}{2 \delta t}\left(f^{n+1}-\frac{4}{3} f^{n}+\frac{1}{3} f^{n-1}\right) .
\end{aligned}
$$

Let us discretize problem (2.5) in time, once $t$ has been normalized. We assume the force term is continuous in time and denote the time level by a superscript. We start using the BDF1 time integration scheme. It leads to the following problem: for $=0,1, \ldots, N-1$, given $u^{n}$, find $u^{n+1} \in \mathcal{V}\left(\Omega_{t^{n+1}}\right)$ such that

$$
\begin{aligned}
& \frac{1}{T}\left(u^{n+1}-u^{n}, v^{n+1}\right)_{\Omega_{t^{n+1}}}+\delta t \nu\left(\nabla u^{n+1}, \nabla v^{n+1}\right)_{\Omega_{t^{n+1}}} \\
& \quad+\delta t\left(\left(\boldsymbol{a}-\boldsymbol{w}^{n+1}\right) \cdot \nabla u^{n+1}, v^{n+1}\right)_{\Omega_{t^{n+1}}}=\delta t\left\langle f^{n+1}, v^{n+1}\right\rangle_{\Omega_{t^{n+1}}}
\end{aligned}
$$

with $u^{0}=u_{0}$ in $L^{2}\left(\Omega_{0}\right)$.

Furthermore, we discretize in time the ALE mapping using a linear interpolation. The discretized ALE mapping $\mathcal{A}_{t}^{n+1}$ is defined for a given time slab $\left[t^{n}, t^{n+1}\right]$ as

$$
\mathcal{A}_{t}^{n+1}\left(x_{0}, t\right)=\frac{t-t^{n}}{\delta t} \mathcal{A}_{t^{n+1}}\left(x_{0}\right)+\frac{t^{n+1}-t}{\delta t} \mathcal{A}_{t^{n}}\left(x_{0}\right) \text {. }
$$

Thus, the mesh velocity is constant on each time step and given by
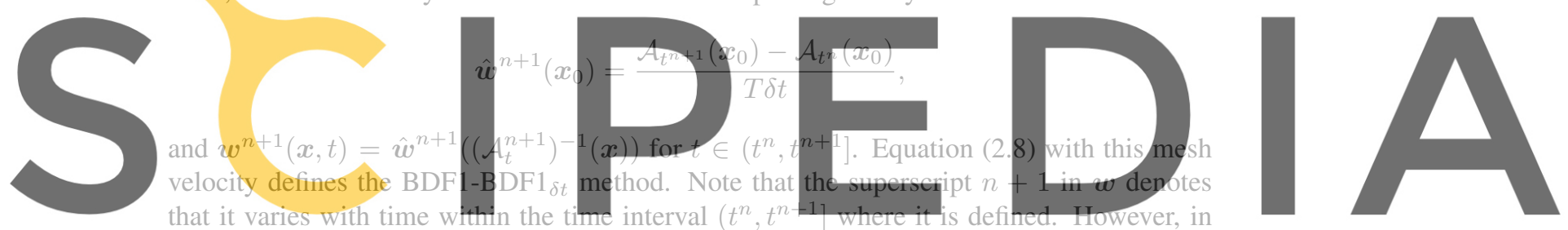

$\S 3$ we will simply denote $w^{n+1} \equiv w^{n+1}\left(x, t^{n+1}\right)$. Since $\mathcal{A}_{t n+1}^{n+1}=\mathcal{A}_{t^{n+1}}$, we will write

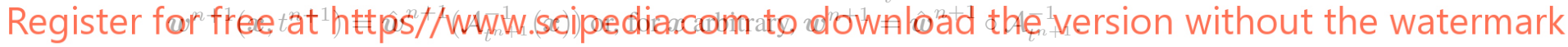

For the numerical analysis we rewrite the transient problem using a different setting.

The sequence of problems (2.8) can be written in a unified manner as: find a sequence $U=$ $\left\{u^{0}, u^{1}, u^{2}, \ldots, u^{N}\right\}$ such that

$$
B(U, V)=L(V)
$$

for all sequences $V$, where

$$
\begin{gathered}
B(U, V):=\frac{1}{2 T}\left(u^{0}, v^{0}\right)_{\Omega_{0}}+\sum_{n=0}^{N-1}\left[\frac{1}{T}\left(\delta u^{n+1}, v^{n+1}\right)_{\Omega_{t^{n+1}}}+\delta t \nu\left(\nabla u^{n+1}, \nabla v^{n+1}\right)_{\Omega_{t^{n+1}}}\right. \\
\left.+\delta t\left(\left(\boldsymbol{a}-\boldsymbol{w}^{n+1}\right) \cdot \nabla u^{n+1}, v^{n+1}\right)_{\Omega_{t^{n+1}}}\right] \\
L(V):=\frac{1}{2 T}\left(u^{0}, v^{0}\right)_{\Omega_{0}}+\sum_{n=0}^{N-1} \delta t\left\langle f^{n+1}, v^{n+1}\right\rangle_{\Omega_{t^{n+1}}}
\end{gathered}
$$

Observe that the initial condition has been embedded in the variational problem. 
In order to reach second order accuracy in time, the BDF2 integration scheme is used. It leads to the following time discretization of (2.5):

$$
\begin{aligned}
& \frac{1}{2 T}\left(3 u^{n+1}-4 u^{n}+u^{n-1}, v^{n+1}\right)_{\Omega_{t^{n+1}}}+\delta t \nu\left(\nabla u^{n+1}, \nabla v^{n+1}\right)_{\Omega_{t^{n+1}}} \\
& \quad+\delta t\left(\left(\boldsymbol{a}-\boldsymbol{w}^{n+1}\right) \cdot \nabla u^{n+1}, v^{n+1}\right)_{\Omega_{t^{n+1}}}=\delta t\left\langle f^{n+1}, v^{n+1}\right\rangle_{\Omega_{t^{n+1}}}
\end{aligned}
$$

This problem has to be initialized. For instance, we can obtain $u^{1}$ with (2.8) and $u^{0}=u_{0}$ in $L^{2}\left(\Omega_{0}\right)$ keeping the order of convergence of the method. In order to keep this accuracy, a quadratic interpolation is used to approximate the ALE mapping. For a given time slab $\left[t^{n}, t^{n+1}\right]$, this interpolation is given by

$$
\begin{aligned}
\mathcal{A}_{t}^{n+1}\left(\boldsymbol{x}_{0}, t\right)= & \frac{\left(t-t^{n}\right)\left(t-t^{n-1}\right)}{2 \delta t^{2}} \mathcal{A}_{t^{n+1}}\left(\boldsymbol{x}_{0}\right) \\
& -\frac{\left(t-t^{n+1}\right)\left(t-t^{n-1}\right)}{2 \delta t^{2}} \mathcal{A}_{t^{n}}\left(\boldsymbol{x}_{0}\right)+\frac{\left(t-t^{n+1}\right)\left(t-t^{n}\right)}{2 \delta t^{2}} \mathcal{A}_{t^{n-1}}\left(\boldsymbol{x}_{0}\right) .
\end{aligned}
$$

Thus, the mesh velocity on each time step is linear in time and given by

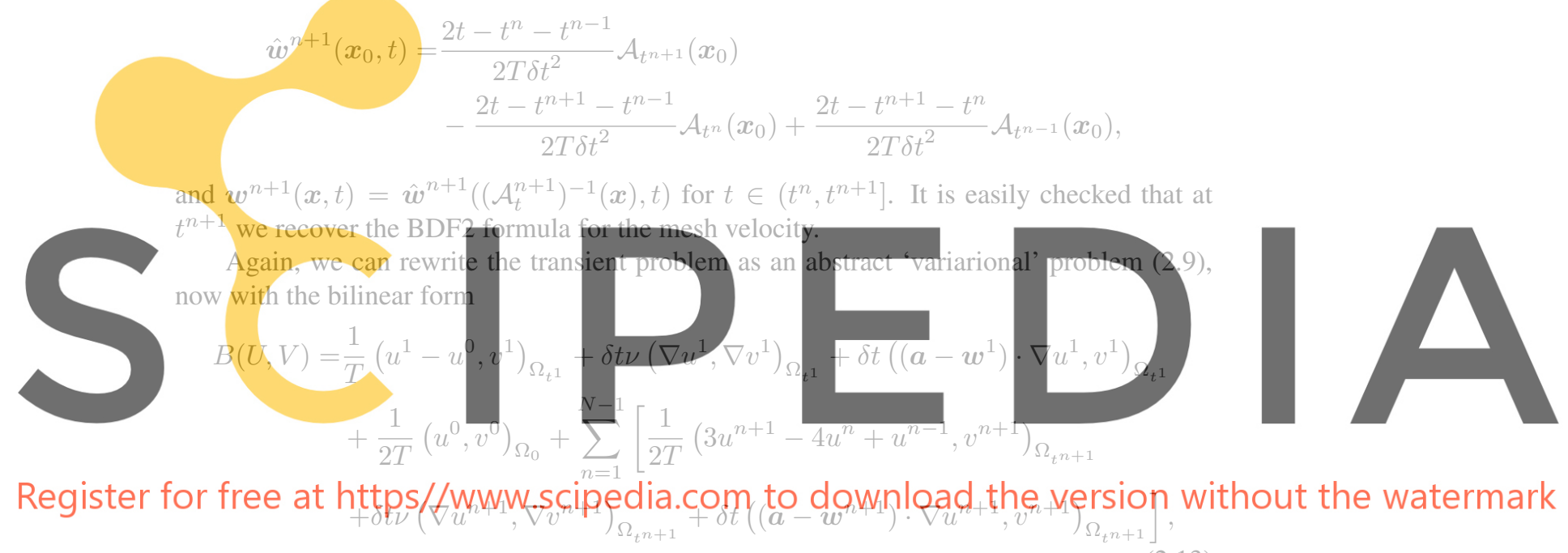

and the linear form

$$
L(V):=\frac{1}{2 T}\left(u^{0}, v^{0}\right)_{\Omega_{0}}+\sum_{n=0}^{N-1} \delta t\left\langle f^{n+1}, v^{n+1}\right\rangle_{\Omega_{t^{n+1}}} .
$$

We end this subsection giving the norm for which stability and convergence results are obtained in $\S 3$ for the previous semi-discrete problems, which is

$$
\left.\|V\|\right|^{2}=\frac{1}{T} \sup _{n \in[0, N]}\left\|v^{n}\right\|_{L^{2}\left(\Omega_{t^{n}}\right)}^{2}+\sum_{n=0}^{N-1} \delta t \nu\left\|\nabla v^{n+1}\right\|_{L^{2}\left(\Omega_{t^{n+1}}\right)}^{2} .
$$

Given a normed space $X$, for $1 \leq q<\infty$ we define the space $\ell^{q}(X)$ as that of sequences $V=\left\{v^{n}\right\}_{n=0}^{N}$ such that $\sum_{n=0}^{N} \delta t\left\|v^{n}\right\|_{X}^{q}<\infty$, and $\ell^{\infty}(X)$ the space of sequences such that $\sup _{n=0, \ldots, N}\left\|v^{n}\right\|_{X}<\infty$. With this notation, the norm defined in (2.15) can be considered that of $\ell^{\infty}\left(L^{2}\left(\Omega_{t}\right)\right) \cap \ell^{2}\left(H_{0}^{1}\left(\Omega_{t}\right)\right)$. Here, the subscript $t$ has to be understood as $t^{n}$ for the $n$-th component of the sequence. 
2.3. The fully discrete problem. At this point we treat the space discretization of systems (2.8) and (2.12). The BDF1-BDF1-OSS $\delta t, h$ reads as follows: for $n=0,1, \ldots, N-1$, given $u_{h}^{n}$, find $u_{h}^{n+1} \in \mathcal{V}_{h}\left(\Omega_{t}\right)$ such that

$$
\begin{aligned}
& \frac{1}{T}\left(u_{h}^{n+1}-u_{h}^{n}, v_{h}^{n+1}\right)_{\Omega_{t^{n+1}}}+\delta t \nu\left(\nabla u_{h}^{n+1}, \nabla v_{h}^{n+1}\right)_{\Omega_{t^{n+1}}} \\
& \quad+\delta t\left(\left(\boldsymbol{a}-\boldsymbol{w}^{n+1}\right) \cdot \nabla u_{h}^{n+1}, v_{h}^{n+1}\right)_{\Omega_{t^{n+1}}} \\
& +\delta t\left(\Pi_{h}^{\perp}\left(\left(\boldsymbol{a}-\boldsymbol{w}^{n+1}\right) \cdot \nabla u_{h}^{n+1}\right), \tau^{n+1}\left(\boldsymbol{a}-\boldsymbol{w}^{n+1}\right) \cdot \nabla v_{h}^{n+1}\right)_{\Omega_{t^{n+1}}} \\
& \quad=\delta t\left\langle f^{n+1}, v_{h}^{n+1}\right\rangle_{\Omega_{t^{n+1}}}
\end{aligned}
$$

where $\mathcal{V}_{h}\left(\Omega_{t}\right)$ is a finite element approximation space of $\mathcal{V}\left(\Omega_{t}\right), \tau^{n+1}$ is a mesh dependent parameter, that we will call stabilization parameter, whose expression is detailed later, and $\Pi_{h}^{\perp}(\cdot)=: I d(\cdot)-\Pi_{h}(\cdot)$, with $I d$ the identity in $L^{2}\left(\Omega_{t}\right)$ and $\Pi_{h}(\cdot)$ the $L^{2}$-projection onto this finite element space (and therefore $\Pi_{h} \frac{\perp}{h}(\cdot)$ is the projection orthogonal to the finite element space). The description and motivation of this formulation, that we call Orthogonal Subgrid Scale (OSS) stabilization, can be found in [9].

Let $\Theta_{h}^{t}$ be a finite element partition of the domain $\Omega_{t}$ in a family of elements $\left\{K_{e}\right\}_{e=1}^{n_{e l}}$, $n_{e l}$ being the number of elements. We denote the diameter of the sphere that circumscribes element $K$ by $h_{K}$ and the diameter of the sphere inscribed in $K$ by $\varrho_{K}$. We also call $h=$ $\max _{K \in \Theta_{h}^{t}}\left(h_{K}\right)$ and $\varrho=\min _{K \in \Theta_{h}^{t}}\left(\varrho_{K}\right)$. We assume that all the element domains $K \in$ $\Theta_{h}^{t}$ are the image of a reference element $\tilde{K}$ through polynomial mappings $F_{K}$, affine for simplicial elements, bilinear for quadrilaterals and trilinear for hexahedra. On $\tilde{K}$ we define the polynomial spaces $R_{p}$ $x_{1}, \ldots, x_{d}$ of degree less than consists of polynomials in called $Q_{p}$. The finite element spaces introduced before are:
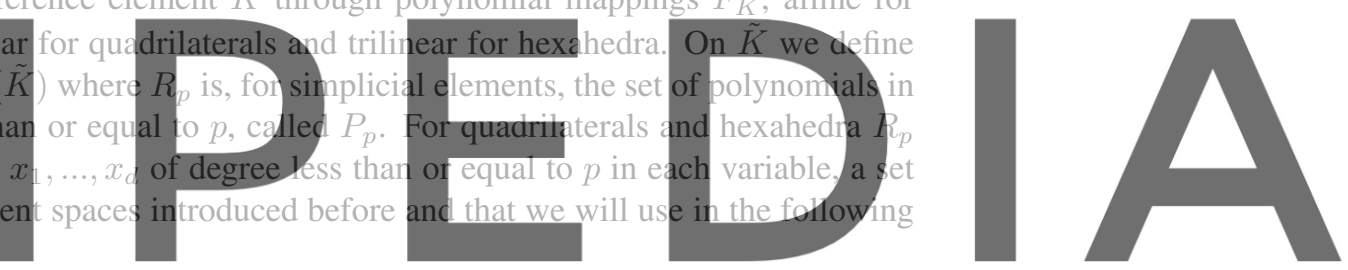

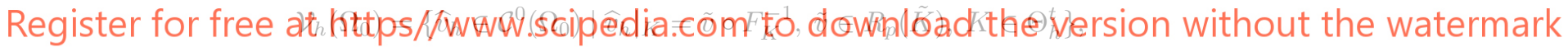

$$
\begin{aligned}
& \mathcal{V}_{h, 0}\left(\Omega_{0}\right)=\left\{v_{h} \in \mathcal{V}_{h}\left(\Omega_{0}\right)\left|v_{h}\right| \partial \Omega_{0}=0\right\}, \\
& \mathcal{V}_{h}\left(\Omega_{t}\right)=\left\{v_{h} \in \mathcal{C}^{0}\left(\Omega_{t}\right) \mid v_{h}=\widehat{v}_{h} \circ \mathcal{A}_{t}^{-1}, \widehat{v}_{h} \in \mathcal{V}_{h}\left(\Omega_{0}\right)\right\}, \\
& \mathcal{V}_{h, 0}\left(\Omega_{t}\right)=\left\{v_{h} \in \mathcal{C}^{0}\left(\Omega_{t}\right) \mid v_{h}=\widehat{v}_{h} \circ \mathcal{A}_{t}^{-1}, \widehat{v}_{h} \in \mathcal{V}_{h, 0}\left(\Omega_{0}\right)\right\} .
\end{aligned}
$$

Moreover, $\Theta_{h}^{t}$ is assumed to be quasi-uniform, that is to say, there exists a constant $\varrho_{2}>0$, independent of $h$, such that $\frac{\varrho}{h} \geq \varrho_{2}>0$ as $h$ tends to zero. This will simplify the analysis and, in particular, will allow us to use stabilization parameters constant in space.

Let us note that in practical applications $\mathcal{A}_{t^{n+1}}$ maps $\Theta_{h}^{0}$ onto $\Theta_{h}^{n+1}$. Therefore, it is easily checked the fact that $\boldsymbol{w}^{n+1} \in\left(\mathcal{V}_{h}\left(\Omega_{t^{n+1}}\right)\right)^{d}$. In the following we will not distinguish between $\boldsymbol{w}^{n+1}$ and $\boldsymbol{w}_{h}^{n+1}$.

Also in this case we can write the problem using a 'variational' formalism. The fully discrete sequence of problems given by (2.16) can be written as: find a sequence $U_{h}=$ $\left\{u_{h}^{0}, u_{h}^{1}, \ldots, u_{h}^{N}\right\}$ such that

$$
B_{h}\left(U_{h}, V_{h}\right)=L\left(V_{h}\right)
$$


for all sequences $V_{h}$, with the bilinear form $B_{h}$ given by

$$
\begin{aligned}
B_{h}\left(U_{h},\right. & \left.V_{h}\right)=\frac{1}{2 T}\left(u_{h}^{0}, v_{h}^{0}\right)_{\Omega_{0}} \\
& +\sum_{n=0}^{N-1}\left[\frac{1}{T}\left(u_{h}^{n+1}-u_{h}^{n}, v_{h}^{n+1}\right)_{\Omega_{t^{n+1}}}+b_{h}\left(\boldsymbol{w}^{n+1} ; u_{h}^{n+1}, v_{h}^{n+1}\right)_{\Omega_{t^{n+1}}}\right],
\end{aligned}
$$

where $b_{h}$ is defined as

$$
\begin{aligned}
& b_{h}\left(\boldsymbol{w}^{n+1} ; u_{h}^{n+1}, v_{h}^{n+1}\right)_{\Omega_{t^{n+1}}}=\delta t \nu\left(\nabla u_{h}^{n+1}, \nabla v_{h}^{n+1}\right)_{\Omega_{t^{n+1}}} \\
& +\delta t\left(\left(\boldsymbol{a}-\boldsymbol{w}^{n+1}\right) \cdot \nabla u_{h}^{n+1}, v_{h}^{n+1}\right)_{\Omega_{t^{n+1}}} \\
& +\delta t\left(\Pi_{h}^{\perp}\left(\left(\boldsymbol{a}-\boldsymbol{w}^{n+1}\right) \cdot \nabla u_{h}^{n+1}\right), \tau^{n+1}\left(\boldsymbol{a}-\boldsymbol{w}^{n+1}\right) \cdot \nabla v_{h}^{n+1}\right)_{\Omega_{t^{n+1}}} .
\end{aligned}
$$

The OSS method modifies the discretized equation of the classical Galerkin method introducing the last term, which enhances the stability of the original method. The value of the stabilization parameter $\tau^{n+1}$ has been justified in [9]. In an ALE framework it depends on the difference between the advection velocity $\boldsymbol{a}$ and the mesh velocity $\boldsymbol{w}$. The expression we use is

$$
\tau^{n+1}=\left(c_{1} \frac{\nu}{h^{2}}+c_{2} \frac{\|\boldsymbol{a}-\boldsymbol{w}\|_{L^{\infty}\left(\Omega_{t^{n+1}}\right)}}{h}\right)^{-1}
$$

that is constant in space. Here, $c_{1}$ and $c_{2}$ are algorithmic constants that depend on the order of the finite element interpolation. As will be shown later (see (4.7)), they are related to the constant $C_{\text {inv }}$ in the inverse estimate introduced in (4.1).

As in [11], we will make further assumptions. We assume that for each $n$ the parameter $\tau^{n}$ satisfies

$$
\tau^{n} \leq C T \delta t
$$

which in particular implies that we can not let $\delta t \rightarrow 0$ without refining the finite element mesh. by

For the space discretization of the second order method (2.12), the bilinear form is given

$$
\begin{aligned}
& B_{h}\left(U_{h}, V_{h}\right) \\
& =\sum_{n=1}^{N-1}\left[\frac{1}{2 T}\left(3 u_{h}^{n+1}-4 u_{h}^{n}+u_{h}^{n-1}, v_{h}^{n+1}\right)_{\Omega_{t^{n+1}}}+b_{h}\left(\boldsymbol{w}^{n+1} ; u_{h}^{n+1}, v_{h}^{n+1}\right)_{\Omega_{t^{n+1}}}\right] \\
& \quad+\frac{1}{T}\left(u_{h}^{1}-u_{h}^{0}, v_{h}^{1}\right)_{\Omega_{t^{1}}}+b_{h}\left(\boldsymbol{w}^{1} ; u_{h}^{1}, v_{h}^{1}\right)_{\Omega_{t^{1}}}+\frac{1}{T}\left(u_{h}^{0}, v_{h}^{0}\right)_{\Omega_{0}} .
\end{aligned}
$$

We end this section with two norms useful in the following numerical analysis. The first is a norm that we will call weak, and given by

$$
\begin{aligned}
\|V\| \|_{w}^{2}= & \frac{1}{T} \sup _{n \in[0, N]}\left\|v^{n}\right\|_{L^{2}\left(\Omega_{t^{n}}\right)}^{2}+\sum_{n=0}^{N-1} \delta t \nu\left\|\nabla v^{n+1}\right\|_{L^{2}\left(\Omega_{t^{n+1}}\right)}^{2} \\
& +\sum_{n=0}^{N-1} \delta t \tau^{n+1}\left\|\Pi_{h}^{\perp}\left(\left(\boldsymbol{a}-\boldsymbol{w}^{n+1}\right) \cdot \nabla v^{n+1}\right)\right\|_{L^{2}\left(\Omega_{t^{n+1}}\right)}^{2}
\end{aligned}
$$


Observe that only the orthogonal projection of the convective term appears. The full convective term appears in the norm that we will call strong, given by

$$
\begin{aligned}
\|V\| \|_{s}^{2}= & \frac{1}{T} \sup _{n \in[0, N]}\left\|v^{n}\right\|_{L^{2}\left(\Omega_{t^{n}}\right)}^{2}+\sum_{n=0}^{N-1} \delta t \nu\left\|\nabla v^{n+1}\right\|_{L^{2}\left(\Omega_{t^{n+1}}\right)}^{2} \\
& +\sum_{n=0}^{N-1} \delta t \tau^{n+1}\left\|\left(\boldsymbol{a}-\boldsymbol{w}^{n+1}\right) \cdot \nabla v^{n+1}\right\|_{L^{2}\left(\Omega_{t^{n+1}}\right)}^{2} \\
= & \|V \mid\|_{w}^{2}+\sum_{n=0}^{N-1} \delta t \tau^{n+1}\left\|\Pi_{h}\left(\left(\boldsymbol{a}-\boldsymbol{w}^{n+1}\right) \cdot \nabla v^{n+1}\right)\right\|_{L^{2}\left(\Omega_{t^{n+1}}\right)}^{2} .
\end{aligned}
$$

3. Analysis of the semi-discrete problem. In this section we analyze problems BDF1$\mathrm{BDF} 1_{\delta t}$ and $\mathrm{BDF} 2-\mathrm{BDF} 2_{\delta t}$. In both cases, stability and error estimates will be given. We denote by $C$ a positive constant, possibly with different values at different appearances.

3.1. Analysis of BDF1-BDF1 $1_{\delta t}$. Let us define by $U_{\text {ex }}=\left\{u_{0}, u\left(t^{1}\right), u\left(t^{2}\right), \ldots, u\left(t^{N}\right)\right\}$ the sequence of solutions of the contiunous problem (2.4) and $U=\left\{u^{0}, u^{1}, u^{2}, \ldots, u^{N}\right\}$ the sequence of solutions of the semi-discrete problem (in time) (2.9)-(2.11). We start obtaining a stability result for this method. With this aim, first we prove that the bilinear form $(2.10)$ that governs the semi-discrete problem is coercive.

THEOREM 3.1 (Coercivity). There exists $\delta t_{\mathrm{cr}}^{1}$ such that for $0<\delta t<\delta t_{\mathrm{cr}}^{1}$ the bilinear form $B(\cdot, \cdot)$ defined in (2.10) is coercive, that is, for every sequence $V=\left\{v^{n}\right\}_{n=0}^{N}$, with $v^{n} \in \mathcal{V}\left(\Omega_{t^{n}}\right)$

$$
B(V, V) \geq \beta_{1}\left|\|V \mid\|^{2}\right.
$$

for a certain constant $\beta_{1} \geq 0$ independent of $h$.

Proof. We know, from the definition of the bilinear form, that

$$
\begin{aligned}
B(V, V)= & \sum_{n=0}^{N-1}\left[\frac{1}{T}\left(v^{n+1}-v^{n}, v^{n+1}\right)_{\Omega_{t^{n+1}}}+\delta t \nu\left\|\nabla v^{n+1}\right\|_{L^{2}\left(\Omega_{t^{n+1}}\right)}^{2}\right. \\
& \left.+\delta t\left(\left(\boldsymbol{a}-\boldsymbol{w}^{n+1}\right) \cdot \nabla v^{n+1}, v^{n+1}\right)_{\Omega_{t^{n+1}}}\right]+\frac{1}{2 T}\left\|v^{0}\right\|_{L^{2}\left(\Omega_{0}\right)}^{2} .
\end{aligned}
$$

We can rewrite the term coming from the time derivative as follows:

$$
\begin{aligned}
& \frac{1}{T}\left(v^{n+1}-v^{n}, v^{n+1}\right)_{\Omega_{t^{n+1}}} \\
& \quad=\frac{1}{2 T}\left[\left\|v^{n+1}\right\|_{L^{2}\left(\Omega_{t^{n+1}}\right)}^{2}-\left\|v^{n}\right\|_{L^{2}\left(\Omega_{t^{n+1}}\right)}^{2}+\left\|v^{n+1}-v^{n}\right\|_{L^{2}\left(\Omega_{t^{n+1}}\right)}^{2}\right] .
\end{aligned}
$$

Integrating equation (2.7) from $t^{n}$ to $t^{n+1}$ for the function $v^{n}$ we get

$$
\frac{1}{T}\left\|v^{n}\right\|_{L^{2}\left(\Omega_{t^{n+1}}\right)}^{2}=\frac{1}{T}\left\|v^{n}\right\|_{L^{2}\left(\Omega_{t^{n}}\right)}^{2}+\int_{t^{n}}^{t^{n+1}} \int_{\Omega_{s}}\left(\nabla \cdot \boldsymbol{w}^{n+1}\right)\left(v^{n}\right)^{2} \mathrm{~d} \Omega \mathrm{d} s,
$$

where we have profited from the fact that the discrete mesh velocity is constant at every time step. On the other hand, due to the fact that the convective velocity $\boldsymbol{a}$ is divergence-free, we get

$$
\begin{aligned}
\left(\left(\boldsymbol{a}-\boldsymbol{w}^{n+1}\right) \cdot \nabla v^{n+1}, v^{n+1}\right)_{\Omega_{t^{n+1}}} & =-\frac{1}{2} \int_{\Omega_{t^{n+1}}} \boldsymbol{w}^{n+1} \cdot \nabla\left(v^{n+1}\right)^{2} \mathrm{~d} \Omega \\
& =\frac{1}{2} \int_{\Omega_{t^{n+1}}}\left(\nabla \cdot \boldsymbol{w}^{n+1}\right)\left(v^{n+1}\right)^{2} \mathrm{~d} \Omega .
\end{aligned}
$$


We bound the terms associated to the mesh velocity as follows:

$$
\begin{aligned}
& \int_{t^{n}}^{t^{n+1}} \int_{\Omega_{s}}\left(\nabla \cdot \boldsymbol{w}^{n+1}\right)\left(v^{n}\right)^{2} \mathrm{~d} \Omega \mathrm{d} s \\
& \leq \delta t \sup _{s \in\left(t^{n}, t^{n+1}\right)}\left\|J_{\mathcal{A}_{t^{n+1}, s}} \nabla \cdot \boldsymbol{w}^{n+1}\right\|_{L^{\infty}\left(\Omega_{s}\right)}\left\|v^{n}\right\|_{L^{2}\left(\Omega_{t^{n+1}}\right)}^{2}, \\
& -\delta t \int_{\Omega_{t^{n+1}}} \boldsymbol{w}^{n+1} \cdot \nabla\left(v^{n+1}\right)^{2} \mathrm{~d} \Omega=\delta t \int_{\Omega_{t^{n+1}}}\left(\nabla \cdot \boldsymbol{w}^{n+1}\right)\left(v^{n+1}\right)^{2} \mathrm{~d} \Omega \\
& \leq \delta t\left\|\nabla \cdot \boldsymbol{w}^{n+1}\right\|_{L^{\infty}\left(\Omega_{t^{n+1}}\right)}\left\|v^{n+1}\right\|_{L^{2}\left(\Omega_{t^{n+1}}\right)}^{2} .
\end{aligned}
$$

Let us define the parameters

$$
\gamma_{1}^{n+1}=T \sup _{s \in\left(t^{n}, t^{n+1}\right)}\left\|J_{\mathcal{A}_{t^{n+1}, s}} \nabla \cdot \boldsymbol{w}^{n+1}\right\|_{L^{\infty}\left(\Omega_{s}\right)},
$$

for $n=-1, \ldots, N-2$ and $\gamma_{1}^{N}=0$, together with

$$
\gamma_{2}^{n+1}=T\left\|\nabla \cdot \boldsymbol{w}^{n}\right\|_{L^{\infty}\left(\Omega_{t^{n+1}}\right)}
$$

for $n=0, \ldots, N-1$ and $\gamma_{2}^{0}=0$.

With the inequalities just proved we can easily obtain that

$$
\begin{aligned}
B(V, V) & +\frac{1}{2 T} \sum_{n=-1}^{N-1} \delta t\left(\gamma_{1}^{n+1}+\gamma_{2}^{n+1}\right)\left\|v^{n+1}\right\|_{L^{2}\left(\Omega_{t^{n+1}}\right)}^{2} \\
& \geq \sup _{n \in[-1, N-1]} \frac{1}{2 T}\left\|v^{n+1}\right\|_{L^{2}\left(\Omega_{t^{n+1}}\right)}^{2}+\sum_{n=0}^{N-1} 2 \delta t \nu\left\|\nabla v^{n+1}\right\|_{L^{2}\left(\Omega_{t^{n+1}}\right)}^{2} .
\end{aligned}
$$

If the maximum of $\left\|v^{n}\right\|_{L^{2}\left(\Omega_{t^{n}}\right)}$ is achieved at $n=N_{m}$, the sequence

$$
\left\{v^{0}, v^{1}, \ldots, v^{N_{m}}, 0, \ldots, 0\right\}
$$

has to be added to the test sequence. Sometimes along the paper we obtain the maximum using this technique. Invoking the Gronwall lemma (see [19]), we can absorb the second term of the left hand side with the first one of the right hand side for a $\delta t$ small enough. More precisely, the time step must be such that

$$
\delta t<\frac{1}{\sup _{n \in[0, N]}\left(\gamma_{1}^{n}+\gamma_{2}^{n}\right)}=: \delta t_{\mathrm{cr}}^{1} .
$$

We note that this is the time step size of the normalized problem in time. The original $\delta t_{\mathrm{cr}}^{1}$ does not depend on $T$ anymore. $\square$

This result, together with the continuity of $L(\cdot)$ proved in next lemma, will lead us to a classical stability bound.

LEMMA 3.2 (Continuity). The following inequality holds:

$$
L(V) \leq \sum_{n=0}^{N-1} \frac{\delta t}{2 \beta \nu}\left\|f^{n+1}\right\|_{H^{-1}\left(\Omega_{t^{n+1}}\right)}^{2}+\sum_{n=0}^{N-1} \frac{\delta t \beta \nu}{2}\left\|\nabla v^{n+1}\right\|_{L^{2}\left(\Omega_{t^{n+1}}\right)}^{2}
$$


for all $\beta>0$.

Proof. The right hand side has the following expression:

$$
L(V)=\sum_{n=0}^{N-1} \delta t\left\langle\boldsymbol{f}^{n+1}, v^{n+1}\right\rangle_{\Omega_{t^{n+1}}} .
$$

The Cauchy-Schwarz inequality leads to

$$
L(V) \leq\left(\sum_{n=0}^{N-1} \frac{\delta t}{\nu}\left\|f^{n+1}\right\|_{H^{-1}\left(\Omega_{t^{n+1}}\right)}^{2}\right)^{\frac{1}{2}}\left(\sum_{n=0}^{N-1} \delta t \nu\left\|\nabla v^{n+1}\right\|_{L^{2}\left(\Omega_{t^{n+1}}\right)}^{2}\right)^{\frac{1}{2}} .
$$

The proof is finished invoking Young's inequality. $\square$

From Theorem 3.1 and Lemma 3.2 the following stability result is straightforward.

COROLlARY 3.3 (Stability). There exists $\delta t_{\mathrm{cr}}^{1}$ such that, for $0<\delta t<\delta t_{\mathrm{cr}}^{1}$, the sequence $U$, solution of problem (2.9)-(2.11), is bounded as follows:

$$
\|\mid U\|^{2} \leq C \sum_{n=0}^{N-1} \frac{\delta t}{\nu}\left\|f^{n+1}\right\|_{H^{-1}\left(\Omega_{t^{n+1}}\right)}^{2} .
$$

REMARK 3.1. The BDF1 method is unconditionally stable for fixed domains. However, for moving domains this property is not maintained anymore. In this case only conditional stability can be proved, with the critical time step value obtained above.

The next task is to obtain an optimal convergence result. In the following theorem, relying on the stability properties proved in Corollary 3.3, optimal error estimates are obtained. We denote by $e^{n+1}:=u\left(t^{n+1}\right)-u^{n+1}$ the error introduced by the time integration at time $t^{n+1}$, and by $E:=U_{\mathrm{ex}}-U$ the sequence of these errors.

THEOREM 3.4 (Convergence). There exists $\delta t_{\mathrm{cr}}^{1}$ such that, for $0<\delta t<\delta t_{\mathrm{cr}}^{1}$, the sequence of errors $E=U_{\mathrm{ex}}-U$ satisfies the following error estimate:

$$
\begin{aligned}
\left\||E \||^{2} \leq C \frac{\delta t^{2}}{T} \sum_{n=0}^{N-1} \delta t\right. & \| \\
+\sup _{s \in\left(t^{n}, t^{n+1}\right)} \| & \left.\left.\left\|\left.\frac{\partial^{2} u}{\partial t^{2}}\right|_{\boldsymbol{x}_{0}}\right\|_{L^{2}\left(\Omega_{t^{n+1}}\right)}^{2}\right|^{2}\left\|_{L^{\infty}\left(\Omega_{0}\right)}\right\| u^{n+1} \|_{H^{1}\left(\Omega_{t^{n+1}}\right)}^{2}\right) .
\end{aligned}
$$

Proof. We start taking the exact solution sequence $U_{\text {ex }}$ in the bilinear form. We get:

$$
\begin{aligned}
B\left(U_{\mathrm{ex}}, V\right)= & L(V)+\sum_{n=0}^{N-1} \frac{1}{T}\left(u\left(t^{n+1}\right)-u\left(t^{n}\right)-\left.\delta t \frac{\partial u}{\partial t}\right|_{t^{n+1}}, v^{n+1}\right)_{\Omega_{t^{n+1}}} \\
& -\sum_{n=0}^{N-1} \delta t\left(\left(\boldsymbol{w}^{n+1}-\boldsymbol{w}\left(t^{n+1}\right)\right) \cdot \nabla u\left(t^{n+1}\right), v^{n+1}\right)_{\Omega_{t^{n+1}}} .
\end{aligned}
$$

We subtract the equation for the semi-discrete sequence of solutions to the previous equations and arrive to

$$
\begin{aligned}
B\left(U-U_{\mathrm{ex}}, V\right)= & -\sum_{n=0}^{N-1} \frac{1}{T}\left(u\left(t^{n+1}\right)-u\left(t^{n}\right)-\left.\delta t \frac{\partial u}{\partial t}\right|_{t^{n+1}}, v^{n+1}\right)_{\Omega_{t^{n+1}}} \\
& +\sum_{n=0}^{N-1} \delta t\left(\left(\boldsymbol{w}^{n+1}-\boldsymbol{w}\left(t^{n+1}\right)\right) \cdot \nabla u\left(t^{n+1}\right), v^{n+1}\right)_{\Omega_{t^{n+1}}} .
\end{aligned}
$$


We test the previous equation with $V=U-U_{\text {ex }}=E$, obtaining

$$
\begin{aligned}
B(E, E)= & -\sum_{n=0}^{N-1} \frac{1}{T}\left(u\left(t^{n+1}\right)-u\left(t^{n}\right)-\left.\delta t \frac{\partial u}{\partial t}\right|_{t^{n+1}}, e^{n+1}\right)_{\Omega_{t^{n+1}}} \\
& +\sum_{n=0}^{N-1} \delta t\left(\left(\boldsymbol{w}^{n+1}-\boldsymbol{w}\left(t^{n+1}\right)\right) \cdot \nabla u\left(t^{n+1}\right), e^{n+1}\right)_{\Omega_{t^{n+1}}} .
\end{aligned}
$$

Exploiting the fact that the bilinear form is coercive the remaining ingredient is an appropriate bound for the error terms associated to the time discretization. Let us start with the terms related to the time derivative. We use the following Taylor formula for $u$ :

$$
\frac{u\left(\boldsymbol{x}_{0}, t^{n+1}\right)-u\left(\boldsymbol{x}_{0}, t^{n}\right)}{T \delta t}-\left.\frac{1}{T} \frac{\partial u}{\partial t}\right|_{\boldsymbol{x}_{0}}\left(t^{n+1}\right)=-\left.\frac{1}{T \delta t} \int_{t^{n}}^{t^{n+1}}\left(s-t^{n}\right) \frac{\partial^{2} u}{\partial t^{2}}\right|_{\boldsymbol{x}_{0}}(s) \mathrm{d} s
$$

and for the mesh velocity

$$
\boldsymbol{w}^{n+1}-\boldsymbol{w}\left(t^{n+1}\right)=-\frac{1}{T \delta t}\left(\int_{t^{n}}^{t^{n+1}}\left(s-t^{n}\right) \frac{\partial^{2} \mathcal{A}_{s}}{\partial t^{2}} \mathrm{~d} s\right) \circ \mathcal{A}_{t^{n+1}}^{-1} .
$$

As explained in $\S 2$, it is understood with this notation that this equality holds for arbitrary $\boldsymbol{x} \in \Omega_{t}$.

With (3.4) we get a bound for the term associated to the time derivative of $u$ as follows:

$$
\begin{aligned}
& \int_{\Omega_{t^{n+1}}} e^{n+1} \cdot\left(\left.\int_{t^{n}}^{t^{n+1}}\left(s-t^{n}\right) \frac{\partial^{2} u}{\partial t^{2}}\right|_{\boldsymbol{x}_{0}}\left(\boldsymbol{x}_{0}, s\right) \mathrm{d} s\right) \circ \mathcal{A}_{t^{n+1}}^{-1} \mathrm{~d} \Omega \\
& \leq \int_{t^{n}}^{t^{n+1}} \int_{\Omega_{0}} J_{\mathcal{A}_{t^{n+1}}}\left(s-t^{n}\right) \widehat{e^{n+1}} \frac{\widehat{\partial^{2} u}}{\partial t^{2}} \mathrm{~d} \Omega \mathrm{d} s \\
& \leq\left(\int_{t^{n}}^{t^{n+1}}\left(s-t^{n}\right)^{2}\left\|e^{n+1}\right\|_{L^{2}\left(\Omega_{t^{n+1}}\right)}^{2}\right)^{\frac{1}{2}} \\
& \quad \times\left(\int_{t^{n}}^{t^{n+1}} \int_{\Omega_{0}} J_{\mathcal{A}_{t^{n+1}}}\left(\frac{\widehat{\partial^{2} u}}{\partial t^{2}}\right)^{2} \mathrm{~d} \Omega \mathrm{d} s\right)^{\frac{1}{2}}, \\
& \leq \frac{\beta_{1} \delta t}{2}\left\|e^{n+1}\right\|_{L^{2}\left(\Omega_{t^{n+1}}\right)}^{2}+C \delta t^{3}\left\|\left.\frac{\partial^{2} u}{\partial t^{2}}\right|_{\boldsymbol{x}_{0}}\right\|_{L^{2}\left(\Omega_{t^{n+1}}\right)}^{2}
\end{aligned}
$$

where $\beta_{1}$ is the coercivity constant introduced in Theorem 3.1. Similarly, using (3.5) for the 
term related to the time derivative of the mapping, we get

$$
\begin{aligned}
& -\int_{\Omega_{t^{n+1}}} e^{n+1}\left(\int_{t^{n}}^{t^{n+1}}\left(s-t^{n}\right) \frac{\partial^{2} \mathcal{A}_{s}}{\partial t^{2}} \mathrm{~d} s\right) \circ \mathcal{A}_{t^{n+1}}^{-1} \cdot \nabla u^{n+1} \mathrm{~d} \Omega \\
& \leq \int_{t^{n}}^{t^{n+1}} \int_{\Omega_{0}} J_{\mathcal{A}_{t^{n+1}}}\left(s-t^{n}\right) \widehat{e^{n+1}} \frac{\partial^{2} \mathcal{A}_{s}}{\partial t^{2}} \cdot \widehat{\nabla u^{n+1}} \mathrm{~d} \Omega \mathrm{d} s \\
& \leq\left(\int_{t^{n}}^{t^{n+1}}\left(s-t^{n}\right)^{2}\left\|e^{n+1}\right\|_{L^{2}\left(\Omega_{t^{n+1}}\right)}^{2} \mathrm{~d} s\right)^{\frac{1}{2}} \\
& \times\left(\int_{t^{n}}^{t^{n+1}}\left\|\left|\frac{\partial^{2} \mathcal{A}_{s}}{\partial t^{2}}\right|^{2}\right\|_{L^{\infty}\left(\Omega_{0}\right)}\left\|u^{n+1}\right\|_{H^{1}\left(\Omega_{t^{n+1}}\right)}^{2} \mathrm{~d} s\right)^{\frac{1}{2}} \\
& \leq \frac{\beta_{1} \delta t}{2}\left\|e^{n+1}\right\|_{L^{2}\left(\Omega_{t^{n+1}}\right)}^{2}+C \delta t^{3} \sup _{s \in\left(t^{n}, t^{n+1}\right)}\left\|\left|\frac{\partial^{2} \mathcal{A}_{s}}{\partial t^{2}}\right|^{2}\right\|_{L^{\infty}\left(\Omega_{0}\right)}\left\|u^{n+1}\right\|_{H^{1}\left(\Omega_{t^{n+1}}\right)}^{2} .
\end{aligned}
$$

With these results we can write

$$
\begin{aligned}
B(E, E) \leq & \frac{1}{T} \sum_{n=0}^{N-1}\left(\delta t \beta_{1}\left\|e^{n+1}\right\|_{L^{2}\left(\Omega_{t^{n+1}}\right)}^{2}+C \delta t^{3}\left\|\left.\frac{\partial^{2} u}{\partial t^{2}}\right|_{\boldsymbol{x}_{0}}\right\|_{L^{2}\left(\Omega_{t^{n+1}}\right)}^{2}\right. \\
& \left.+C \delta t^{3} \sup _{s \in\left(t^{n}, t^{n+1}\right)}\left\|\left|\frac{\partial^{2} \mathcal{A}_{s}}{\partial t^{2}}\right|^{2}\right\|_{L^{\infty}\left(\Omega_{0}\right)}\left\|u^{n+1}\right\|_{H^{1}\left(\Omega_{t^{n+1}}\right)}^{2}\right) .
\end{aligned}
$$

At this point we invoke the coercivity property of the bilinear form proved in Theorem 3.1. Thus, the first term of the right hand side in (3.6) can be absorbed using the Gronwall lemma . We note that in this case we can apply the Gronwall lemma without any extra condition over the time step size (see [19]).

Clearly, the second term in the right-hand-side of (3.3) is bounded if the second time derivatives of the ALE mapping are uniformly bounded in $[0, T]$. In this case, its norm in the space $L^{\infty}\left(0, T ; L^{\infty}\left(\Omega_{0}\right)\right)$ can be taken out of the sum and the stability estimate of Corollary 3.3 allows us to bound the remaining term. However, we have kept expression (3.3) to display the structure of the error bound.

We conclude this subsection with an improved stability estimate:

COROLLARY 3.5 (Stability in $\ell^{\infty}\left(H^{2}\left(\Omega_{t}\right)\right)$ ). Under the conditions of Theorem 3.4, suppose additionally that the right-hand-side of (3.3) is bounded, that $u \in L^{\infty}\left(0, T ; H^{2}\left(\Omega_{t}\right)\right)$ and that the domain $\Omega_{t}$ is such that $\Delta u \in L^{2}\left(\Omega_{t}\right)$ implies $u \in H^{2}\left(\Omega_{t}\right)$. Then, $U \in$ $\ell^{\infty}\left(H^{2}\left(\Omega_{t}\right)\right)$.

Proof. At each time step we can write the error equation

$$
\begin{aligned}
\nu \Delta\left(u^{n+1}-u\left(t^{n+1}\right)\right) & =\left(\boldsymbol{a}-\boldsymbol{w}^{n+1}\right) \cdot \nabla\left(u^{n+1}-u\left(t^{n+1}\right)\right) \\
& +\left(\boldsymbol{w}\left(t^{n+1}\right)-\boldsymbol{w}^{n+1}\right) \cdot \nabla u\left(t^{n+1}\right)+\frac{1}{\delta t}\left(u^{n+1}-u^{n}\right)-\left.\frac{\partial u}{\partial t}\right|_{t^{n+1}} .
\end{aligned}
$$

By virtue of Theorem 3.4, all the terms in the right-hand-side are bounded in $L^{2}\left(\Omega_{t^{n+1}}\right)$ for $n=0, \ldots, N-1$. Since

$$
\left\|\Delta u^{n+1}\right\|_{L^{2}\left(\Omega_{t^{n+1}}\right)} \leq\left\|\Delta u^{n+1}-\Delta u\left(t^{n+1}\right)\right\|_{L^{2}\left(\Omega_{t^{n+1}}\right)}+\left\|\Delta u\left(t^{n+1}\right)\right\|_{L^{2}\left(\Omega_{t^{n+1}}\right)},
$$


it follows that $\left\{\Delta u^{n+1}\right\}_{n=0}^{N-1} \in \ell^{\infty}\left(L^{2}\left(\Omega_{t}\right)\right)$. The assumption on the domain $\Omega_{t}$ implies that $\left\{u^{n+1}\right\}_{n=0}^{N-1} \in \ell^{\infty}\left(H^{2}\left(\Omega_{t}\right)\right)$

This justifies our strategy of first analyzing the problem semi-discretized in time and then the fully discrete problem. When we will require $U \in \ell^{2}\left(H^{p+1}\left(\Omega_{t}\right)\right)$ to obtain optimal order of convergence in space, we know that at least for $p=1$ this holds under the same condition on the domain $\Omega_{t}$ as for the sequence of solutions of the continuous problem, $U_{\mathrm{ex}}$. It is well known that this condition on $\Omega_{t}$ holds for example if it is convex and polyhedral (see, for example, [17]).

3.2. Analysis of BDF2-BDF $2_{\delta t}$. For the second order method we follow the same procedure used above. In this case the problem that we analyze can be written using equation (2.9) together with the bilinear form (2.13) and the right hand side linear form (2.14), and we denote by $U=\left\{u^{0}, u^{1}, u^{2}, \ldots, u^{N}\right\}$ the sequence of solutions of this problem.

We start again proving that the corresponding bilinear form is coercive.

THEOREM 3.6 (Coercivity). There exists $\delta t_{\mathrm{cr}}^{2}$ such that for $0<\delta t<\delta t_{\mathrm{cr}}^{2}$ the bilinear form $B(\cdot, \cdot)$ defined in (2.12) is coercive, that is, for every sequence $V=\left\{v^{n}\right\}_{n=0}^{N}$, with $v^{n} \in \mathcal{V}\left(\Omega_{t^{n}}\right)$,

$$
B(V, V) \geq \beta_{2} \mid\|V\|^{2}
$$

for a certain constant $\beta_{2} \geq 0$ independent of $h$.

Proof. We know, from the definition of the bilinear form, that,

$$
\begin{aligned}
B(V, V)= & \sum_{n=0}^{N-1}\left[\delta t\left\|\nabla v^{n+1}\right\|_{L^{2}\left(\Omega_{t^{n+1}}\right)}^{2}+\delta t\left(\left(\boldsymbol{a}-\boldsymbol{w}^{n+1}\right) \cdot \nabla v^{n+1}, v^{n+1}\right)_{\Omega_{t^{n+1}}}\right] \\
& +\sum_{n=1}^{N-1} \frac{1}{2 T}\left(3 v^{n+1}-4 v^{n}+v^{n-1}, v^{n+1}\right)_{\Omega_{t^{n+1}}}+\frac{1}{T}\left(v^{1}-v^{0}, v^{1}\right)_{\Omega_{t^{1}}} \\
& +\frac{1}{2 T}\left\|v^{0}\right\|_{L^{2}\left(\Omega_{0}\right)}^{2} .
\end{aligned}
$$

Integrating equation (2.7) from $t^{n}$ to $t^{n+1}$ for the functions $v^{n}$ and $2 v^{n}-v^{n-1}$, we can express the term corresponding to discrete time derivative as follows:

$$
\begin{aligned}
& \frac{1}{2 T}\left(3 v^{n+1}-4 v^{n}+v^{n-1}, 4 v^{n+1}\right)_{\Omega_{t^{n+1}}} \\
& =\frac{1}{T}\left(\left\|v^{n+1}\right\|_{L^{2}\left(\Omega_{t^{n+1}}\right)}^{2}-\left\|v^{n}\right\|_{L^{2}\left(\Omega_{t^{n}}\right)}^{2}+\left\|2 v^{n+1}-v^{n}\right\|_{L^{2}\left(\Omega_{t^{n+1}}\right)}^{2}\right. \\
& \left.\quad-\left\|2 v^{n}-v^{n-1}\right\|_{L^{2}\left(\Omega_{t^{n}}\right)}^{2}+\left\|\delta^{2} v^{n+1}\right\|_{L^{2}\left(\Omega_{t^{n+1}}\right)}^{2}\right) \\
& \quad+\int_{t^{n}}^{t^{n+1}} \int_{\Omega_{s}}\left(\nabla \cdot \boldsymbol{w}^{n+1}(s)\right)\left(v^{n}\right)^{2} \mathrm{~d} \Omega \mathrm{d} s \\
& \quad+\int_{t^{n}}^{t^{n+1}} \int_{\Omega_{s}}\left(\nabla \cdot \boldsymbol{w}^{n+1}(s)\right)\left(2 v^{n}-v^{n-1}\right)^{2} \mathrm{~d} \Omega \mathrm{d} s
\end{aligned}
$$


The mesh velocity terms are bounded as follows:

$$
\begin{gathered}
\int_{t^{n}}^{t^{n+1}} \int_{\Omega_{s}}\left(\nabla \cdot \boldsymbol{w}^{n+1}(s)\right)\left(v^{n}\right)^{2} \mathrm{~d} \Omega \mathrm{d} s+\int_{t^{n}}^{t^{n+1}} \int_{\Omega_{s}}\left(\nabla \cdot \boldsymbol{w}^{n+1}(s)\right)\left(2 v^{n}-v^{n-1}\right)^{2} \mathrm{~d} \Omega \mathrm{d} s \\
\leq \delta t \sup _{s \in\left(t^{n}, t^{n+1}\right)}\left\|J_{\mathcal{A}_{t^{n+1}, s}} \nabla \cdot \boldsymbol{w}^{n+1}(s)\right\|_{L^{\infty}\left(\Omega_{s}\right)} \\
\times\left(\left\|v^{n}\right\|_{L^{2}\left(\Omega_{t^{n+1}}\right)}^{2}+\left\|2 v^{n}-v^{n-1}\right\|_{L^{2}\left(\Omega_{t^{n+1}}\right)}^{2}\right) .
\end{gathered}
$$

On the other hand, we can exploit the fact that the convective velocity $\boldsymbol{a}$ is divergence-free, obtaining for the convective term that

$$
\begin{aligned}
\left(\left(\boldsymbol{a}-\boldsymbol{w}^{n+1}\right) \cdot \nabla v^{n+1}, 4 v^{n+1}\right)_{\Omega_{t^{n+1}}} & =-2 \delta t \int_{\Omega_{t^{n+1}}} \boldsymbol{w}^{n+1} \cdot \nabla\left(v^{n+1}\right)^{2} \mathrm{~d} \Omega \\
& =2 \delta t \int_{\Omega_{t^{n+1}}}\left(\nabla \cdot \boldsymbol{w}^{n+1}\right)\left(v^{n+1}\right)^{2} \mathrm{~d} \Omega \\
& \leq 2\left\|\nabla \cdot \boldsymbol{w}^{n+1}\right\|_{L^{\infty}\left(\Omega_{t^{n+1}}\right)}\left\|u_{h}^{n+1}\right\|_{L^{2}\left(\Omega_{t^{n+1}}\right)}^{2} .
\end{aligned}
$$

We use inequalities (3.8) and (3.9) in (3.7) and invoke again the Gronwall lemma. This leads to the desired bound for a time step size:

$$
\delta t<\frac{1}{\sup _{n \in[0, N]}\left(\gamma_{1}^{n}+2 \gamma_{2}^{n}\right)}=: \delta t_{\mathrm{cr}}^{2},
$$

slightly different from the one obtained for the first order method. $\square$

The previous theorem and Lemma 3.2 allow us to obtain the same stability result as for the previous case, stated in the next corollary.

COROLLARY 3.7 (Stability). There exists $\delta t_{\mathrm{cr}}^{2}$ such that for $0<\delta t<\delta t_{\mathrm{cr}}^{2}$ the sequence $U$ solution of problem (2.9)-(2.13)-(2.14) is bounded as follows:

$$
\|U\|\left\|^{2} \leq C \sum_{n=0}^{N-1} \frac{\delta t}{\nu}\right\| f^{n+1} \|_{H^{-1}\left(\Omega_{t^{n+1}}\right)}^{2} .
$$

Furthermore, we can obtain optimal error estimates under some regularity assumptions. For the sake of clearness we assume that the initialization is calculated exactly. It can be easily checked from Theorem 3.4 that the error introduced by the initialization is optimal.

THEOREM 3.8 (Convergence). There exist $\delta t_{\mathrm{cr}}^{2}$ such that for $0<\delta t<\delta t_{\mathrm{cr}}^{2}$ the sequence of errors $E=U_{e x}-U$ satisfies the following error estimate:

$$
\begin{aligned}
\|E\| \|^{2} \leq C \frac{\delta t^{4}}{T} \sum_{n=0}^{N-1} \delta t & \| \\
+ & \left.\frac{\partial^{3} u}{\partial t^{3}}\right|_{\boldsymbol{x}_{0}} \|_{L^{2}\left(\Omega_{t^{n+1}}\right)}^{2} \\
& \left.\sup _{s \in\left(t^{n}, t^{n+1}\right)}\left\|\left|\frac{\partial^{3} \mathcal{A}_{s}}{\partial t^{3}}\right|^{2}\right\|\left\|_{L^{\infty}\left(\Omega_{0}\right)}\right\| u^{n+1} \|_{H^{1}\left(\Omega_{t^{n+1}}\right)}^{2}\right)
\end{aligned}
$$


Proof. We start taking the exact solution sequence $U_{e x}$ in the bilinear form. We get:

$$
\begin{aligned}
B\left(U_{e x}, V\right)= & L(V)+\sum_{n=0}^{N-1} \frac{1}{2 T}\left(3 u\left(t^{n+1}\right)-4 u\left(t^{n}\right)+u\left(t^{n-1}\right)-\left.\delta t \frac{\partial u}{\partial t}\right|_{t^{n+1}}, v^{n+1}\right)_{\Omega_{t^{n+1}}} \\
& -\sum_{n=0}^{N-1} \delta t\left(\left(\boldsymbol{w}^{n+1}-\boldsymbol{w}\left(t^{n+1}\right)\right) \cdot \nabla u\left(t^{n+1}\right), v^{n+1}\right)_{\Omega_{t^{n+1}}} .
\end{aligned}
$$

Now we subtract the equation for the semi-discrete sequence of solutions to the previous equations and arrive to

$$
\begin{aligned}
B\left(U-U_{e x}, V\right)= & -\sum_{n=0}^{N-1} \frac{1}{T}\left(3 u\left(t^{n+1}\right)-4 u\left(t^{n}\right)+u\left(t^{n-1}\right)-\left.\delta t \frac{\partial u}{\partial t}\right|_{t^{n+1}}, v^{n+1}\right)_{\Omega_{t^{n+1}}} \\
& +\sum_{n=0}^{N-1} \delta t\left(\left(\boldsymbol{w}^{n+1}-\boldsymbol{w}\left(t^{n+1}\right)\right) \cdot \nabla u\left(t^{n+1}\right), v^{n+1}\right)_{\Omega_{t^{n+1}}} .
\end{aligned}
$$

We test the previous equation with $V=U-U_{e x}=E$, obtaining

$$
\begin{aligned}
B(E, E)= & -\sum_{n=0}^{N-1} \frac{1}{T}\left(3 u\left(t^{n+1}\right)-4 u\left(t^{n}\right)+u\left(t^{n-1}\right)-\left.\delta t \frac{\partial u}{\partial t}\right|_{t^{n+1}}, e^{n+1}\right)_{\Omega_{t^{n+1}}} \\
& +\sum_{n=0}^{N-1} \delta t\left(\left(\boldsymbol{w}^{n+1}-\boldsymbol{w}\left(t^{n+1}\right)\right) \cdot \nabla u\left(t^{n+1}\right), e^{n+1}\right)_{\Omega_{t^{n+1}}} .
\end{aligned}
$$

The truncation error introduced by the time integration scheme BDF2 is evaluated using the following Taylor formula:

$$
\begin{aligned}
& \frac{3 u\left(\boldsymbol{x}_{0}, t^{n+1}\right)-4 u\left(\boldsymbol{x}_{0}, t^{n}\right)+u\left(\boldsymbol{x}_{0}, t^{n-1}\right)}{T \delta t}-\left.\frac{1}{T} \frac{\partial u}{\partial t}\right|_{\boldsymbol{x}_{0}}\left(t^{n+1}\right) \\
& =-\left.\frac{1}{T \delta t} \int_{t^{n-1}}^{t^{n+1}}\left(s-t^{n}\right)^{2} \frac{\partial^{3} u}{\partial t^{3}}\right|_{\boldsymbol{x}_{0}}(s) \mathrm{d} s-\left.\frac{1}{T \delta t} \int_{t^{n}}^{t^{n+1}}\left(s-t^{n}\right)^{2} \frac{\partial^{3} u}{\partial t^{3}}\right|_{\boldsymbol{x}_{0}}(s) \mathrm{d} s .
\end{aligned}
$$

The evaluation of the mesh velocity (2.6) requires a time derivative. Its numerical approximation using the second order BDF2 scheme can be written again as a truncation error:

$$
\begin{aligned}
\boldsymbol{w}^{n+1} & -\boldsymbol{w}\left(t^{n+1}\right) \\
= & -\frac{1}{T \delta t}\left(\int_{t^{n}-1}^{t^{n+1}}\left(s-t^{n}\right)^{2} \frac{\partial^{3} \mathcal{A}_{s}}{\partial t^{3}} \mathrm{~d} s+\int_{t^{n}}^{t^{n+1}}\left(s-t^{n}\right)^{2} \frac{\partial^{3} \mathcal{A}_{s}}{\partial t^{3}} \mathrm{~d} s\right) \circ \mathcal{A}_{t^{n+1}}^{-1},
\end{aligned}
$$

which holds for all $\boldsymbol{x} \in \Omega_{t}$. Recall that $\boldsymbol{w}^{n+1}$ stands for the mesh velocity evalutated at $t^{n+1}$.

The error related to the time derivative of $u$ can be bounded using the following inequal- 
ity:

$$
\begin{aligned}
& \int_{\Omega_{t^{n+1}}} e^{n+1} \cdot\left(\left.\int_{t^{n-1}}^{t^{n+1}}\left(s-t^{n}\right)^{2} \frac{\partial^{3} u}{\partial t^{3}}\right|_{\boldsymbol{x}_{0}}(s) \mathrm{d} s\right. \\
& \left.-\left.\frac{1}{T \delta t} \int_{t^{n}}^{t^{n+1}}\left(s-t^{n}\right)^{2} \frac{\partial^{3} u}{\partial t^{3}}\right|_{\boldsymbol{x}_{0}}(s) \mathrm{d} s\right) \circ \mathcal{A}_{t^{n+1}}^{-1} \mathrm{~d} \Omega \\
& \leq \frac{\beta_{2} \delta t}{2}\left\|e^{n+1}\right\|_{L^{2}\left(\Omega_{t^{n+1}}\right)}^{2}+C \delta t^{5}\left\|\left.\frac{\partial^{2} u}{\partial t^{2}}\right|_{\boldsymbol{x}_{0}}\right\|_{L^{2}\left(\Omega_{t^{n+1}}\right)}^{2},
\end{aligned}
$$

where $\beta_{2}$ is the coercivity constant introduced in Theorem 3.6.

We obtain the following inequality in order to bound the error introduced by the evaluation of the mesh velocity,

$$
\begin{aligned}
& -\int_{\Omega_{t^{n+1}}} e^{n+1}\left(\int_{t^{n}-1}^{t^{n+1}}\left(s-t^{n}\right)^{2} \frac{\partial^{3} \mathcal{A}_{s}}{\partial t^{3}} \mathrm{~d} s\right. \\
& \left.\quad+\int_{t^{n}}^{t^{n+1}}\left(s-t^{n}\right)^{2} \frac{\partial^{3} \mathcal{A}_{s}}{\partial t^{3}} \mathrm{~d} s\right) \circ \mathcal{A}_{t^{n+1}}^{-1} \cdot \nabla u^{n+1} \mathrm{~d} \Omega \\
& \leq \frac{\beta_{2} \delta t}{2}\left\|e^{n+1}\right\|_{L^{2}\left(\Omega_{t^{n+1}}\right)}^{2}+\delta t^{5} \sup _{s \in\left(t^{n-1}, t^{n+1}\right)}\left\|\left|\frac{\partial^{3} \mathcal{A}_{s}}{\partial t^{3}}\right|^{2}\right\|_{L^{\infty}\left(\Omega_{0}\right)}\left\|u^{n+1}\right\|_{H^{1}\left(\Omega_{t^{n+1}}\right)}^{2} .
\end{aligned}
$$

Using the error expressions (3.11) and (3.12) and bounds (3.13) and (3.14) we get

$$
\begin{aligned}
B(E, E) \leq & \frac{1}{T} \sum_{n=0}^{N-1} \delta t \beta_{2}\left\|e^{n+1}\right\|_{L^{2}\left(\Omega_{t^{n+1}}\right)}^{2}+C \frac{\delta t^{4}}{T} \sum_{n=0}^{N-1} \delta t\left\|\left.\frac{\partial^{3} u}{\partial t^{3}}\right|_{\boldsymbol{x}_{0}}\right\|_{L^{2}\left(\Omega_{t^{n+1}}\right)}^{2} \\
& +C \frac{\delta t^{4}}{T} \sum_{n=0}^{N-1} \delta t \sup _{s \in\left(t^{n}, t^{n+1}\right)}\left\|\left|\frac{\partial^{3} \mathcal{A}_{s}}{\partial t^{3}}\right|^{2}\right\|_{L^{\infty}\left(\Omega_{0}\right)}\left\|u^{n+1}\right\|_{H^{1}\left(\Omega_{t^{n+1}}\right)}^{2} .
\end{aligned}
$$

Again, we can apply the Gronwall lemma without any extra condition over the time step size.

4. The fully discrete problem. In this section we analyze the fully discrete problems $\mathrm{BDF} 1-\mathrm{BDF} 1-\mathrm{OSS}_{\delta t, h}$ and BDF2-BDF2-OSS ${ }_{\delta t, h}$. In both cases, stability and error estimates are obtained.

Observe from (2.20) that $\tau^{n}$ has been taken constant in space. Further, we assume $\Theta_{h}^{t}$ quasi-uniform. In this case, the following inverse estimate holds (see [4]):

$$
\left\|\nabla v_{h}\right\|_{L^{2}\left(\Omega_{t}\right)} \leq \frac{C_{\mathrm{inv}}}{h}\left\|v_{h}\right\|_{L^{2}\left(\Omega_{t}\right)} .
$$

In order to obtain optimal convergence results, we assume that $u^{n+1} \in H^{p+1}\left(\Omega_{t}\right)$ for $n=0, \ldots, N-1$, where $p$ is the degree of the polynomial defining the finite element space $\mathcal{V}_{h}$. We also assume that for any function $v \in H^{p+1}\left(\Omega_{t}\right)$ there exists a finite element interpolation $\pi_{h}(v)$ such that,

$$
\left\|v-\pi_{h}(v)\right\|_{H^{m}\left(\Omega_{t}\right)} \leq C_{h} h^{p+1-m}\|v\|_{H^{p+1}\left(\Omega_{t}\right)} .
$$


We need to prove that the $L^{2}$-projection onto the finite element space is an optimal interpolation in the $L^{2}\left(\Omega_{t}\right)$ norm and the seminorm $\|\nabla(\cdot)\|_{L^{2}\left(\Omega_{t}\right)}$. We show this in the following lemma.

LEMMA 4.1. Given a function $v \in H^{p+1}\left(\Omega_{t}\right)$ with $p \geq 1$, its $L^{2}$-projection onto the finite element space $\Pi_{h}(v)$ satisfies

$$
\left\|v-\Pi_{h}(v)\right\|_{L^{2}\left(\Omega_{t}\right)} \leq C_{h} h^{p+1}\|v\|_{H^{p+1}\left(\Omega_{t}\right)},
$$

and also

$$
h^{2}\left\|\Delta v-\Pi_{h}(\Delta v)\right\|_{L^{2}\left(\Omega_{t}\right)} \leq C h^{p+1}\|v\|_{H^{p+1}\left(\Omega_{t}\right)} .
$$

If the inverse estimate (4.1) hold true

$$
\left\|\nabla\left(v-\Pi_{h}(v)\right)\right\|_{L^{2}\left(\Omega_{t}\right)} \leq C h^{p}\|v\|_{H^{p+1}\left(\Omega_{t}\right)},
$$

are satisfied.

Proof. The first inequality is obvious:

$$
\left\|v-\Pi_{h}(v)\right\|_{L^{2}\left(\Omega_{t}\right)} \leq\left\|v-\pi_{h}(v)\right\|_{L^{2}\left(\Omega_{t}\right)} \leq C_{h} h^{p+1}\|v\|_{H^{p+1}\left(\Omega_{t}\right)} .
$$

Using the previous inequality for $\Delta v$ we obtain the second result.

Making use of the inverse estimate (4.1) we prove the last inequality:

$$
\begin{aligned}
\left\|\nabla\left(v-\Pi_{h}(v)\right)\right\|_{L^{2}\left(\Omega_{t}\right)} & \leq\left\|\nabla\left(v-\pi_{h}(v)\right)\right\|_{L^{2}\left(\Omega_{t}\right)}+\left\|\nabla\left(\pi_{h}(v)-\Pi_{h}(v)\right)\right\|_{L^{2}\left(\Omega_{t}\right)} \\
& =\left\|\nabla\left(v-\pi_{h}(v)\right)\right\|_{L^{2}\left(\Omega_{t}\right)}+\left\|\nabla\left(\Pi_{h}\left(v-\pi_{h}(v)\right)\right)\right\|_{L^{2}\left(\Omega_{t}\right)} \\
& \leq\left\|\nabla\left(v-\pi_{h}(v)\right)\right\|_{L^{2}\left(\Omega_{t}\right)}+\frac{C_{\mathrm{inv}}}{h}\left\|\Pi_{h}\left(v-\pi_{h}(v)\right)\right\|_{L^{2}\left(\Omega_{t}\right)} \\
& \leq\left(1+C_{\mathrm{inv}}\right) C_{h} h^{p}\|v\|_{H^{p+1}\left(\Omega_{t}\right)} .
\end{aligned}
$$

As in the previous section, $C$ is a positive constant, possibly with different values at different appearances.

4.1. Analysis of BDF1-BDF1-OSS $\delta t, h$. In this subsection we analyze the fully discrete problem (2.17) with the bilinear form $B_{h}(\cdot, \cdot)$ defined in (2.18) and right-hand side (2.14). We denote by $U=\left\{u^{0}, u^{1}, u^{2}, \ldots, u^{N}\right\}$ the sequence of solutions of the semi-discrete problem (in time) (2.9)-(2.11) and $U_{h}=\left\{u_{h}^{0}, u_{h}^{1}, u_{h}^{2}, \ldots, u_{h}^{N}\right\}$ its fully discrete counterpart, solution of (2.17)-(2.18)-(2.14).

We start proving the coercivity of the bilinear form for the weak norm $\left|\left\|\cdot|\||_{w}\right.\right.$. This result will be used in the convergence analysis.

THEOREM 4.2 (Coercivity). There exists $\delta t_{\mathrm{cr}}^{1}$ such that for $0<\delta t<\delta t_{\mathrm{cr}}^{1}$ the bilinear form $B_{h}(\cdot, \cdot)$ defined in (2.18) is coercive. That is, for every sequence $V=\left\{v^{n}\right\}_{n=0}^{N}$, with $v^{n} \in \mathcal{V}\left(\Omega_{t^{n}}\right)$,

$$
B_{h}(V, V) \geq \beta_{1}|||V| \|_{w}^{2}
$$

for a certain constant $\beta_{1}>0$. 
Proof. The bilinear form analyzed in this theorem is equal to the one for which coercivity is proved in Theorem 3.1 plus the stabilization term. We can easily get

$$
\begin{aligned}
& B_{h}(V, V)=\frac{1}{2 T}\left\|v^{N}\right\|_{L^{2}\left(\Omega^{N}\right)}^{2}+\frac{1}{2 T} \sum_{n=0}^{N-1}\left\|\delta v^{n+1}\right\|_{L^{2}\left(\Omega_{t^{n+1}}\right)}^{2} \\
& \quad+\sum_{n=0}^{N-1}\left[\delta t \nu\left\|\nabla v^{n+1}\right\|_{L^{2}\left(\Omega_{t^{n+1}}\right)}^{2}+\delta t \tau^{n+1}\left\|\Pi_{h}^{\perp}\left(\left(\boldsymbol{a}-\boldsymbol{w}^{n+1}\right) \cdot \nabla v^{n+1}\right)\right\|_{L^{2}\left(\Omega_{t^{n+1}}\right)}^{2}\right] \\
& \quad+\frac{1}{2} \sum_{n=0}^{N-1} \delta t\left(\nabla \cdot \boldsymbol{w}^{n+1},\left(v^{n+1}\right)^{2}\right)_{\Omega_{t^{n+1}}}+\frac{1}{2} \sum_{n=0}^{N-1} \int_{t^{n}}^{t^{n+1}} \int_{\Omega_{t}}\left(\nabla \cdot \boldsymbol{w}^{n+1}\right)\left(v^{n+1}\right)^{2} \mathrm{~d} \Omega .
\end{aligned}
$$

Due to the fact that the stabilization term does not affect the treatment of the mesh velocity terms in Theorem 3.1, we refer to this one for the remaining of the proof. $\square$

Let us define the $\Lambda$-coercivity property associated to a bilinear form that will be used in the following analysis.

Definition 4.3 ( $\Lambda$-coercivity). Let $\mathcal{V}$ be a functional space and $\zeta: \mathcal{V} \times \mathcal{V} \longrightarrow \mathbb{R}$ a bilinear form. We say that $\zeta$ is $\Lambda$-coercive with respect to the norm \|\|$\cdot \|$ and the linear operator $\Lambda: \mathcal{V} \longrightarrow \mathcal{V}$ if there exists and a constant $\beta>0$ such that,

$$
\zeta(v, \Lambda(v)) \geq \beta \mid\|v\| \|^{2}, \quad \forall v \in \mathcal{V} .
$$

The bilinear form $\zeta(\cdot, \cdot)$ also satisfies an inf-sup condition under the conditions of the following lemma.

LEMMA 4.4. If $\Lambda$ is continuous with respect to the norm $\|\cdot\| \cdot \|$ and $\zeta(\cdot, \cdot)$ is $\Lambda$-coercive, then there exists $\gamma>0$ such that

$$
\inf _{u \in \mathcal{V}} \sup _{v \in \mathcal{V}} \frac{\zeta(u, v)}{\||| u|\||\|v\||} \geq \gamma
$$

The proof of the previous lemma is straightforward from Definition 4.3 and the continuity of the operator $\Lambda(\cdot)$.

We now show that the bilinear form $B_{h}(\cdot, \cdot)$ of our problem is $\Lambda$-coercive for the strong norm $\||| \cdot \mid\|_{s}$.

THEOREM 4.5 ( $\Lambda$-coercivity). Let $V=\left\{v^{n}\right\}_{n=0}^{N}$ be a sequence of functions such that $v^{n} \in \mathcal{V}\left(\Omega_{t^{n}}\right)$ and consider the operator

$$
\Lambda(V)=V+\left\{0, \frac{1}{2}\left\{\tau^{n+1} \Pi_{h}\left(\left(\boldsymbol{a}-\boldsymbol{w}^{n+1}\right) \cdot \nabla v^{n+1}\right)\right\}_{0}^{N-1}\right\}
$$

Then, there exists $\delta t_{\mathrm{cr}}^{1}$ such that, for $0<\delta t<\delta t_{\mathrm{cr}}^{1}$, the bilinear form $B_{h}(\cdot, \cdot)$ is $\Lambda$-coercive:

$$
B_{h}(V, \Lambda(V)) \geq \beta_{1}\||V|\|_{s}^{2}
$$

for a certain constant $\beta_{1}>0$.

Proof. Testing (2.18) with the sequence of functions that belong to the finite element space

$$
\Pi_{0}(\tau, V):=\left\{0,\left\{\tau^{n+1} \Pi_{0}\left(v^{n+1}\right)\right\}\right\}_{n=0}^{N-1}:=\left\{0,\left\{\tau^{n+1} \Pi_{h}\left(\left(\boldsymbol{a}-\boldsymbol{w}^{n+1}\right) \cdot \nabla v^{n+1}\right)\right\}_{n=0}^{N-1}\right\}
$$


we have

$$
\begin{aligned}
B_{h}\left(V, \Pi_{0}(\tau, V)\right) \geq & \sum_{n=0}^{N-1} \phi^{n+1} \delta t \tau^{n+1}\left\|\Pi_{h}\left(\left(\boldsymbol{a}-\boldsymbol{w}^{n+1}\right) \cdot \nabla v^{n+1}\right)\right\|_{L^{2}\left(\Omega_{t^{n+1}}\right)}^{2} \\
& -\sum_{n=0}^{N-1}\left[\frac{1}{T}\left\|\delta v^{n+1}\right\|_{L^{2}\left(\Omega_{t^{n+1}}\right)}^{2}+\delta t \nu\left\|\nabla v^{n+1}\right\|_{L^{2}\left(\Omega_{t^{n+1}}\right)}^{2}\right. \\
& \left.+\delta t \tau^{n+1}\left\|\Pi_{h}^{\perp}\left(\left(\boldsymbol{a}-\boldsymbol{w}^{n+1}\right) \cdot \nabla v^{n+1}\right)\right\|_{L^{2}\left(\Omega_{t^{n+1}}\right)}^{2}\right]
\end{aligned}
$$

where

$$
\phi^{n+1}:=1-\frac{1}{4} \frac{\tau^{n+1}}{T \delta t}-\frac{1}{4} \tau^{n+1} \frac{\nu C_{\mathrm{inv}}^{2}}{h^{2}}-\frac{1}{4}\left(\tau^{n+1}\right)^{2} \frac{\left\|\boldsymbol{a}-\boldsymbol{w}^{n+1}\right\|_{L^{\infty}\left(\Omega_{t^{n+1}}\right)}^{2} C_{\mathrm{inv}}^{2}}{h^{2}} .
$$

To obtain (4.7) we have made use of Young's inequality and the inverse estimate (4.1). Assuming now that the constants $c_{1}$ and $c_{2}$ in (2.20) are such that $c_{1} \leq C_{\mathrm{inv}}^{2}, c_{2} \leq C_{\mathrm{inv}}$ and the constant $C$ in (2.21) is $C \leq 1$ it follows that $\phi^{n+1} \geq 1 / 4$.

The combination of (4.7) and (4.5) leads to

$$
\begin{aligned}
B_{h}(V, 2 V & \left.+\Pi_{0}(\tau, V)\right) \geq \frac{1}{T}\left\|v^{N}\right\|_{L^{2}\left(\Omega^{N}\right)}^{2}+\sum_{n=0}^{N-1} \delta t \nu\left\|\nabla v^{n+1}\right\|_{L^{2}\left(\Omega_{t^{n+1}}\right)}^{2} \\
& +C \sum_{n=0}^{N-1} \delta t \tau^{n+1}\left\|\left(\boldsymbol{a}-\boldsymbol{w}^{n+1}\right) \cdot \nabla v^{n+1}\right\|_{L^{2}\left(\Omega_{t^{n+1}}\right)}^{2} \\
& +\sum_{n=0}^{N-1} \delta t\left(\nabla \cdot \boldsymbol{w}^{n+1},\left(v^{n+1}\right)^{2}\right)_{\Omega_{t^{n+1}}}+\sum_{n=0}^{N-1} \int_{t^{n}}^{t^{n+1}} \int_{\Omega_{s}}\left(\nabla \cdot \boldsymbol{w}^{n+1}\right)\left(v^{n+1}\right)^{2} \mathrm{~d} \Omega \mathrm{d} s \\
\geq & \frac{1}{T}\left\|v^{N}\right\|_{L^{2}\left(\Omega^{N}\right)}^{2}+\sum_{n=0}^{N-1} \delta t \nu\left\|\nabla v^{n+1}\right\|_{L^{2}\left(\Omega_{t^{n+1}}\right)}^{2} \\
& +C \sum_{n=0}^{N-1} \delta t \tau^{n+1}\left\|\Pi_{h}\left(\left(\boldsymbol{a}-\boldsymbol{w}^{n+1}\right) \cdot \nabla v^{n+1}\right)\right\|_{L^{2}\left(\Omega_{t^{n+1}}\right)}^{2} \\
& -\sum_{n=0}^{N-1} \delta t \gamma_{n+1}\left\|v^{n+1}\right\|_{L^{2}\left(\Omega_{t^{n+1}}\right)}^{2}
\end{aligned}
$$

with $\gamma_{n+1}:=\gamma_{1}^{n+1}+\gamma_{2}^{n+1}$, and $\gamma_{1}^{n+1}, \gamma_{2}^{n+1}$ defined in (3.1) and (3.2). Using the Gronwall lemma, we finally get the coercivity stated in the theorem. We point out that the critical time step $\delta t_{\mathrm{cr}}^{1}$ in this case is identical to the one obtained for the semi-discrete problem.

In order to satisfy the continuity of $\Lambda(\cdot)$ needed for to obtain the inf-sup condition in Lemma 4.4 we have to restrict the situation to the discrete finite element space $\mathcal{V}_{h}$.

LEMMA 4.6 (Continuity). Let $V_{h}=\left\{v_{h}^{n}\right\}_{n=0}^{N}$ be a finite element sequence such that $v_{h}^{n} \in \mathcal{V}_{h}\left(\Omega_{t^{n}}\right)$ and consider the operator $\Lambda$ introduced in Theorem 4.5. Then, $\Lambda(\cdot)$ is continuous with respect to the norm $\left.\||\cdot|\|\right|_{s}$ for every finite element sequence $V_{h}$ :

$$
\left\|\Lambda \Lambda\left(V_{h}\right)\right\|_{s} \leq \rho\left\|\mid V_{h}\right\|_{s}
$$

for a certain constant $\rho>0$. 
Proof. Defining $\Pi_{0}\left(\tau, V_{h}\right)$ as in the proof of the previous theorem, we have from the definition of the norm that

$$
\begin{aligned}
\|\| \Pi_{0}\left(\tau, V_{h}\right)\|\|_{s}^{2}= & \frac{1}{T} \sup _{n \in[0, N-1]}\left\|\tau^{n+1} \Pi_{0}\left(v_{h}^{n+1}\right)\right\|_{L^{2}\left(\Omega_{t^{n+1}}\right)}^{2} \\
& +\sum_{n=0}^{N-1} \delta t \nu\left\|\tau^{n+1} \nabla \Pi_{0}\left(v_{h}^{n+1}\right)\right\|_{L^{2}\left(\Omega_{t^{n+1}}\right)}^{2} \\
& +\sum_{n=0}^{N-1} \delta t \tau^{n+1}\left\|\tau^{n+1}\left(\boldsymbol{a}-\boldsymbol{w}^{n+1}\right) \cdot \nabla \Pi_{0}\left(v_{h}^{n+1}\right)\right\|_{L^{2}\left(\Omega_{t^{n+1}}\right)}^{2} .
\end{aligned}
$$

Invoking the expression for $\tau^{n+1}$ and the inverse estimate (4.1) we can easily bound every term by $\||V|\|_{s}^{2} . \square$

REMARK 4.1. The fact that we need to use the inverse estimate (4.1) in order to bound the first term in (4.10) restricts the continuity of $\Lambda(\cdot)$ to finite element sequences (for the rest of the terms the inverse estimate is applied to derivatives of $\Pi_{h}\left(\left(\boldsymbol{a}-\boldsymbol{w}^{n+1}\right) \cdot \nabla v^{n+1}\right)$, a finite element function even if $v^{n+1}$ is not in the finite element space). However, this restriction does not complicate the convergence analysis, where only the $\Lambda$-coercivity is invoked.

From Lemmas 4.4 and 4.6 we obtain the discrete inf-sup condition.

COROLlaRY 4.7 (Discrete inf-sup condition). Let $U_{h}=\left\{u_{h}^{n}\right\}_{n=0}^{N}$ and $V_{h}=\left\{v_{h}^{n}\right\}_{n=0}^{N}$ be sequences of finite element functions such that $u^{n}, v^{n} \in \mathcal{V}\left(\Omega_{t^{n}}\right)$. There exists $\delta t_{\text {cr }}^{1}$ such that, for $0<\delta t<\delta t_{\mathrm{cr}}^{1}$, the bilinear form $B_{h}(\cdot, \cdot)$ satisfies the following condition:

$$
\inf _{U_{h} \in \mathcal{V}_{h}} \sup _{V_{h} \in \mathcal{V}_{h}} \frac{B_{h}\left(U_{h}, V_{h}\right)}{\left\|\left.U_{h}||\right|_{s}|| \mid V_{h}\right\|_{s}} \geq \widetilde{\beta}_{1}
$$

for a certain constant $\widetilde{\beta}_{1}>0$.

At this point, the only ingredient that remains in order to have a stability result is the continuity of the force term, provided by Lemma 3.2. The stability result is stated in the next corollary.

COROLlARY 4.8 (Stability). There exists $\delta t_{\mathrm{cr}}^{1}$ such that, for $0<\delta t<\delta t_{\mathrm{cr}}^{1}$, the sequence $U_{h}$, solution of problem (2.17)-(2.18)-(2.11), is bounded as follows:

$$
\left\|\mid U_{h}\right\|_{s}^{2} \leq C \sum_{n=0}^{N-1} \frac{\delta t}{\nu}\left\|f^{n+1}\right\|_{H^{-1}\left(\Omega_{t^{n+1}}\right)}^{2} .
$$

For the convergence analysis, let us define the difference between the solution of (2.8) and (2.16) as $e_{d}^{n+1}:=u_{h}^{n+1}-u^{n+1}$, and the sequence of these errors by $E_{d}$. From Theorem 4.5, that proves the $\Lambda$-coercivity of the bilinear form $B_{h}$ for $\Lambda$ defined in this theorem, we know that

$$
B_{h}\left(E_{d}, \Lambda\left(E_{d}\right)\right) \geq \beta_{1}\left\|\mid E_{d}\right\|_{s}^{2}
$$

We subtract the discrete bilinear form (2.18) from its semi-discrete counterpart (2.10) tested with finite element sequences in order to get

$$
\begin{aligned}
& B_{h}\left(E_{d}, V_{h}\right)=\epsilon_{c}\left(V_{h}\right) \\
& \quad:=-\sum_{n=0}^{N-1} \delta t \tau^{n+1}\left(\Pi_{h}^{\perp}\left(\left(\boldsymbol{a}-\boldsymbol{w}^{n+1}\right) \cdot \nabla u^{n+1}\right),\left(\boldsymbol{a}-\boldsymbol{w}^{n+1}\right) \cdot \nabla v_{h}^{n+1}\right)_{\Omega_{t^{n+1}}},
\end{aligned}
$$


where $\epsilon_{c}\left(V_{h}\right)$ accounts for the consistency error. After some manipulations, we can write

$$
\begin{aligned}
B_{h}\left(E_{d}, \Lambda\left(E_{d}\right)\right) & =B_{h}\left(E_{d}, E_{d}\right)+\frac{1}{2} B_{h}\left(E_{d}, \Pi_{0}\left(\tau, E_{d}\right)\right) \\
& =B_{h}\left(E_{d}, \Pi_{h}(U)-U\right)+\epsilon_{c}\left(U_{h}-\Pi_{h}(U)\right)+\frac{1}{2} \epsilon_{c}\left(\Pi_{0}\left(\tau, E_{d}\right)\right),
\end{aligned}
$$

where $\Pi_{h}(U):=\left\{\Pi_{h}\left(u^{n}\right)\right\}_{n=0}^{N}$.

We distinguish between interpolation error, the first term of the right hand side, and the consistency error associated to the second and third terms. In the following two lemmas we bound these error terms. We start with the interpolation error, obtaining the result stated in the following lemma:

LEMMA 4.9 (Interpolation error). The error sequence $E_{d}=U_{h}-U$ satisfies the following inequality:

$$
B_{h}\left(E_{d}, \Pi_{h}(U)-U\right) \leq C \mid\left\|E_{d}\right\| \|_{w}\left(h^{2(p+1)} \sum_{n=0}^{N-1} \delta t\left(\tau^{n+1}\right)^{-1}\left\|u^{n+1}\right\|_{H^{p+1}\left(\Omega_{t^{n+1}}\right)}^{2}\right)^{\frac{1}{2}} .
$$

Proof. Let us expand the expression of the interpolation error making use of the definition of the bilinear form associated to the problem we are analyzing:

$$
\begin{aligned}
B_{h} & \left(E_{d}, \Pi_{h}(U)-U\right) \\
& =\sum_{n=0}^{N-1}\left[\frac{1}{T}\left(e_{d}^{n+1}-e_{d}^{n}, \Pi_{h}\left(u^{n+1}\right)-u^{n+1}\right)_{\Omega_{t^{n+1}}}\right. \\
& +\delta t \nu\left(\nabla e_{d}^{n+1}, \nabla\left(\Pi_{h}\left(u^{n+1}\right)-u^{n+1}\right)\right)_{\Omega_{t^{n+1}}} \\
& +\delta t\left(\left(\boldsymbol{a}-\boldsymbol{w}^{n+1}\right) \cdot \nabla e_{d}^{n+1}, \Pi_{h}\left(u^{n+1}\right)-u^{n+1}\right)_{\Omega_{t^{n+1}}} \\
& \left.+\delta t \tau^{n+1}\left(\Pi_{h}^{\perp}\left(\left(\boldsymbol{a}-\boldsymbol{w}^{n+1}\right) \cdot \nabla e_{d}^{n+1}\right),\left(\boldsymbol{a}-\boldsymbol{w}^{n+1}\right) \cdot \nabla\left(\Pi_{h}\left(u^{n+1}\right)-u^{n+1}\right)\right)_{\Omega_{t^{n+1}}}\right] .
\end{aligned}
$$

We must control each term separately. Let us start with the discrete time derivative term. Using assumption (2.21) we have that

$$
\begin{aligned}
\sum_{n=0}^{N-1} \frac{1}{T}\left(e_{d}^{n+1}-e_{d}^{n}, \Pi_{h}\left(u^{n+1}\right)-u^{n+1}\right)_{\Omega_{t^{n+1}}} \\
\leq C\left(\sum_{n=0}^{N-1} \frac{1}{T}\left\|e_{d}^{n+1}-e_{d}^{n}\right\|_{L^{2}\left(\Omega_{t^{n+1}}\right)}^{2}\right)^{\frac{1}{2}} \\
\quad \times\left(h^{2(p+1)} \sum_{n=0}^{N-1} \delta t\left(\tau^{n+1}\right)^{-1}\left\|u^{n+1}\right\|_{H^{p+1}\left(\Omega_{t^{n+1}}\right)}^{2}\right)^{\frac{1}{2}} .
\end{aligned}
$$

For the viscosity term, using the definition of $\tau^{n+1}$ and the inverse estimate (4.5), we have that

$$
\begin{aligned}
& \sum_{n=0}^{N-1} \delta t \nu\left(\nabla e_{d}^{n+1}, \nabla\left(\Pi_{h}\left(u^{n+1}\right)-u^{n+1}\right)\right)_{\Omega_{t^{n+1}}} \\
& \leq C\left(\sum_{n=0}^{N-1} \delta t \nu\left\|\nabla e_{d}^{n+1}\right\|_{L^{2}\left(\Omega_{t^{n+1}}\right)}^{2}\right)^{\frac{1}{2}} \times\left(h^{2(p+1)} \sum_{n=0}^{N-1} \delta t\left(\tau^{n+1}\right)^{-1}\left\|u^{n+1}\right\|_{H^{p+1}\left(\Omega_{t^{n+1}}\right)}^{2}\right)^{\frac{1}{2}} .
\end{aligned}
$$


Similar arguments allow us to obtain a bound for the convective term:

$$
\begin{aligned}
\sum_{n=0}^{N-1} \delta t & \left(\left(\boldsymbol{a}-\boldsymbol{w}^{n+1}\right) \cdot \nabla e_{d}^{n+1}, \Pi_{h}\left(u^{n+1}\right)-u^{n+1}\right)_{\Omega_{t^{n+1}}} \\
\leq C & \left(\sum_{n=0}^{N-1} \delta t \tau^{n+1}\left\|\Pi_{h}^{\perp}\left(\left(\boldsymbol{a}-\boldsymbol{w}^{n+1}\right) \cdot \nabla e_{d}^{n+1}\right)\right\|_{L^{2}\left(\Omega_{t^{n+1}}\right)}^{2}\right)^{\frac{1}{2}} \\
& \times\left(h^{2(p+1)} \sum_{n=0}^{N-1} \delta t\left(\tau^{n+1}\right)^{-1}\left\|u^{n+1}\right\|_{H^{p+1}\left(\Omega_{t^{n+1}}\right)}^{2}\right.
\end{aligned}
$$

and for the stabilization term we obtain

$$
\begin{aligned}
& \sum_{n=0}^{N-1} \delta t \tau^{n+1}\left(\Pi_{h}^{\perp}\left(\left(\boldsymbol{a}-\boldsymbol{w}^{n+1}\right) \cdot \nabla e_{d}^{n+1}\right),\left(\boldsymbol{a}-\boldsymbol{w}^{n+1}\right) \cdot \nabla\left(\Pi_{h}\left(u^{n+1}\right)-u^{n+1}\right)\right)_{\Omega_{t^{n+1}}} \\
& \leq C\left(\sum_{n=0}^{N-1} \delta t \tau^{n+1}\left\|\Pi_{h}^{\perp}\left(\left(\boldsymbol{a}-\boldsymbol{w}^{n+1}\right) \cdot \nabla e_{d}^{n+1}\right)\right\|_{L^{2}\left(\Omega_{t^{n+1}}\right)}^{2}\right)^{\frac{1}{2}} \\
& \times\left(h^{2(p+1)} \sum_{n=0}^{N-1} \delta t\left(\tau^{n+1}\right)^{-1}\left\|u^{n+1}\right\|_{H^{p+1}\left(\Omega_{t^{n+1}}\right)}^{2} \cdot\right.
\end{aligned}
$$

All the terms have been bounded by the right-hand-side of (4.12), and therefore the proof is finished. $\square$

REMARK 4.2. Invoking the interpolation error (4.2) in (4.13) has allowed us to obtain an optimal bound for the interpolation error without the control of the full convective term in the norm ||$|\cdot| \|_{w}$. This fact will be used for the analysis of the second order method.

The following lemma is devoted to the control of the consistency error. Since we are interested in smooth solutions, say $u \in L^{2}\left(0, T ; H^{p+1}\left(\Omega_{t}\right)\right.$ ) (with the obvious modifications for $u$ less regular), we assume that $f$ is also smooth, in particular $f \in L^{2}\left(0, T ; H^{p-1}\left(\Omega_{t}\right)\right)$. Thus, for $p \geq 1,\left\langle f, v_{h}\right\rangle_{\Omega_{t}}=\left(\Pi_{h}(f), v_{h}\right)_{\Omega_{t}}$. Therefore, the finite element solution is not altered if we assume $\Pi_{h}^{\perp}(f)=0$.

LEMMA 4.10 (Consistency error). The following inequality holds:

$$
\begin{aligned}
\epsilon_{c}\left(U_{h}\right. & \left.-\Pi_{h}(U)+\frac{1}{2} \Pi_{0}\left(\tau, E_{d}\right)\right) \leq C\left(h^{2(p+1)} \sum_{n=0}^{N-1} \delta t\left(\tau^{n+1}\right)^{-1}\left\|u^{n+1}\right\|_{H^{p+1}\left(\Omega_{t^{n+1}}\right)}^{2}\right)^{\frac{1}{2}} \\
& \times\left(\left\|\mid E_{d}\right\|\left\|_{s}^{2}+h^{2(p+1)} \sum_{n=0}^{N-1} \delta t\left(\tau^{n+1}\right)^{-1}\right\| u^{n+1} \|_{H^{p+1}\left(\Omega_{t^{n+1}}\right)}^{2}\right)^{\frac{1}{2}}
\end{aligned}
$$


Proof. From the expression of the consistency error we arrive to:

$$
\begin{aligned}
& -\epsilon_{c}\left(U_{h}-\Pi_{h}(U)\right) \\
& =\sum_{n=0}^{N-1} \delta t \tau^{n+1}\left(\Pi_{h}^{\perp}\left(\left(\boldsymbol{a}-\boldsymbol{w}^{n+1}\right) \cdot \nabla u^{n+1}\right),\left(\boldsymbol{a}-\boldsymbol{w}^{n+1}\right) \cdot \nabla\left(u_{h}^{n+1}-\Pi_{h}\left(u^{n+1}\right)\right)\right)_{\Omega_{t^{n+1}}} \\
& =\sum_{n=0}^{N-1} \delta t \tau^{n+1}\left(\Pi_{h}^{\perp}\left(\left(\boldsymbol{a}-\boldsymbol{w}^{n+1}\right) \cdot \nabla u^{n+1}\right),\left(\boldsymbol{a}-\boldsymbol{w}^{n+1}\right) \cdot \nabla e_{d}^{n+1}\right)_{\Omega_{t^{n+1}}} \\
& +\sum_{n=0}^{N-1} \delta t \tau^{n+1}\left(\Pi_{h}^{\perp}\left(\left(\boldsymbol{a}-\boldsymbol{w}^{n+1}\right) \cdot \nabla u^{n+1}\right),\left(\boldsymbol{a}-\boldsymbol{w}^{n+1}\right) \cdot \nabla\left(u^{n+1}-\Pi_{h}\left(u^{n+1}\right)\right)\right)_{\Omega_{t^{n+1}}} .
\end{aligned}
$$

On the other hand, from the equation for the semi-discrete unknown (2.8), we can easily check that

$$
\begin{aligned}
& \left(\Pi_{h}^{\perp}\left(\left(\boldsymbol{a}-\boldsymbol{w}^{n+1}\right) \cdot \nabla u^{n+1}\right), v^{n+1}\right)_{\Omega_{t^{n+1}}} \\
& \quad=\left(\Pi_{h}^{\perp}\left(\nu \Delta u^{n+1}-\frac{1}{T \delta t}\left(u^{n+1}-u^{n}\right)\right), v^{n+1}\right)_{\Omega_{t^{n+1}}} \\
& \quad=:\left(\Pi_{h}^{\perp}\left(\lambda\left(u^{n+1}\right)\right), v^{n+1}\right)_{\Omega_{t^{n+1}}}
\end{aligned}
$$

where $\lambda(\cdot):=\nu \Delta(\cdot)-\frac{\delta(\cdot)}{T \delta t}$. Note that we have not included $\Pi_{h}^{\perp}(f)$ in the previous equation.

Now, using (4.16) in (4.15) we can split the error in two different terms bounded as follows:

$$
\begin{aligned}
\sum_{n=0}^{N-1} \delta t \tau^{n+1}\left(\Pi_{h}^{\perp}\left(\lambda\left(u^{n+1}\right)\right),\left(\boldsymbol{a}-\boldsymbol{w}^{n+1}\right) \cdot \nabla e_{d}^{n+1}\right)_{\Omega_{t^{n+1}}} \\
\leq C\left(\sum_{n=0}^{N-1} \delta t \tau^{n+1}\left\|\Pi_{h}^{\perp}\left(\lambda\left(u^{n+1}\right)\right)\right\|_{L^{2}\left(\Omega_{t^{n+1}}\right)}^{2}\right)^{\frac{1}{2}} \\
\times\left(\sum_{n=0}^{N-1} \delta t \tau^{n+1}\left\|\Pi_{h}^{\perp}\left(\left(\boldsymbol{a}-\boldsymbol{w}^{n+1}\right) \cdot \nabla e_{d}^{n+1}\right)\right\|_{L^{2}\left(\Omega_{t^{n+1}}\right)}^{2}\right)^{\frac{1}{2}}, \\
\sum_{n=0}^{N-1} \delta t \tau^{n+1}\left(\Pi_{h}^{\perp}\left(\lambda\left(u^{n+1}\right)\right),\left(\boldsymbol{a}-\boldsymbol{w}^{n+1}\right) \cdot \nabla\left(u^{n+1}-\Pi_{h}\left(u^{n+1}\right)\right)\right)_{\Omega_{t^{n+1}}} \\
\leq C\left(\sum_{n=0}^{N-1} \delta t \tau^{n+1}\left\|\Pi_{h}^{\perp}\left(\lambda\left(u^{n+1}\right)\right)\right\|_{L^{2}\left(\Omega_{t^{n+1}}\right)}^{2}\right)^{\frac{1}{2}} \\
\times\left(h^{2(p+1)} \sum_{n=0}^{N-1} \delta t\left(\tau^{n+1}\right)^{-1}\left\|u^{n+1}\right\|_{H^{p+1}\left(\Omega_{t^{n+1}}\right)}^{2}\right)^{\frac{1}{2}} .
\end{aligned}
$$

On the other hand, the term related to the perturbation of the test function $\Pi_{0}\left(\tau, E_{d}\right)$ appear- 
ing in (4.14) can be bounded using similar arguments, leading to

$$
\begin{aligned}
& \epsilon_{c}\left(\Pi_{0}\left(\tau, E_{d}\right)\right) \\
& =\sum_{n=0}^{N-1} \delta t \tau^{n+1}\left(\Pi_{h}^{\perp}\left(\lambda\left(u^{n+1}\right)\right),\left(\boldsymbol{a}-\boldsymbol{w}^{n+1}\right) \cdot \nabla\left(\tau^{n+1} \Pi_{h}\left(\left(\boldsymbol{a}-\boldsymbol{w}^{n+1}\right) \cdot \nabla e_{d}^{n+1}\right)\right)\right)_{\Omega_{t^{n+1}}} \\
& \leq C\left(\sum_{n=0}^{N-1} \delta t \tau^{n+1}\left\|\Pi_{h}^{\perp}\left(\lambda\left(u^{n+1}\right)\right)\right\|_{L^{2}\left(\Omega_{t^{n+1}}\right)}^{2}\right)^{\frac{1}{2}} \\
& \quad \times\left(\sum_{n=0}^{N-1} \delta t \tau^{n+1}\left\|\Pi_{h}\left(\left(\boldsymbol{a}-\boldsymbol{w}^{n+1}\right) \cdot \nabla e_{d}^{n+1}\right)\right\|_{L^{2}\left(\Omega_{t^{n+1}}\right)}^{2}\right)^{\frac{1}{2}} \cdot
\end{aligned}
$$

It only remains to prove that

$$
\sum_{n=0}^{N-1} \delta t \tau^{n+1}\left\|\Pi_{h}^{\perp}\left(\lambda\left(u^{n+1}\right)\right)\right\|_{L^{2}\left(\Omega_{t^{n+1}}\right)}^{2} \leq C h^{2(p+1)} \sum_{n=0}^{N-1} \delta t\left(\tau^{n+1}\right)^{-1}\left\|u^{n+1}\right\|_{H^{p+1}\left(\Omega_{t^{n+1}}\right)}^{2} .
$$

This inequality can be easily obtained from the expression of $\tau^{n+1}$, assumption (2.19) and the interpolation error estimate (4.3). $\square$

We end this section with the main convergence result, which is a direct consequence of inequality (4.11), Lemma 4.9 and Lemma 4.10:

THEOREM 4.11 (Convergence). There exist $\delta t_{\mathrm{cr}}^{1}$ such that, for $0<\delta t<\delta t_{\mathrm{cr}}^{1}$, the sequence of errors $E_{d}=U_{h}-U$ satisfies the following error estimate:

$$
\left\|E_{d}\right\|_{s}^{2} \leq C h^{2(p+1)} \sum_{n=0}^{N-1} \delta t\left(\tau^{n+1}\right)^{-1}\left\|u^{n+1}\right\|_{H^{p+1}\left(\Omega_{t^{n+1}}\right)}^{2} .
$$

4.2. Analysis of BDF2-BDF2-OSS B $_{\delta, h}$. In this subsection we analyze the fully discrete problem (2.17) with the bilinear form $B_{h}(\cdot, \cdot)$ defined in (2.22) and right-hand side (2.14). We denote by $U=\left\{u^{0}, u^{1}, u^{2}, \ldots, u^{N}\right\}$ the sequence of solutions of the second order semidiscrete problem (in time) (2.9)-(2.13)-(2.14) and $U_{h}=\left\{u_{h}^{0}, u_{h}^{1}, u_{h}^{2}, \ldots, u_{h}^{N}\right\}$ its fully discrete counterpart, solution of (2.17)-(2.22)-(2.14).

We have obtained the results of this section using the weak norm $\left.|||\cdot|\right|_{w}$. Let us start with a theorem proving coercivity under the weaker norm.

THEOREM 4.12 (Coercivity). There exists $\delta t_{\mathrm{cr}}^{2}$ such that, for $0<\delta t<\delta t_{\mathrm{cr}}^{2}$, the bilinear form $B_{h}(\cdot, \cdot)$ defined in (2.22) is coercive. That is, for every sequence $V=\left\{v^{n}\right\}_{n=0}^{N}$ with $v^{n} \in V\left(\Omega_{t^{n}}\right)$

$$
B_{h}(V, V) \geq \beta_{2}\|\| V \|_{w}^{2}
$$

for a certain constant $\beta_{2}>0$.

Proof. It can be easily shown that

$$
\begin{aligned}
& B_{h}(V, 4 V) \geq \frac{1}{T}\left(\left\|v^{N}\right\|_{L^{2}\left(\Omega^{N}\right)}^{2}+\sum_{n=0}^{N-1}\left\|\delta^{2} v^{n+1}\right\|_{L^{2}\left(\Omega_{t^{n+1}}\right)}^{2}\right) \\
& +\sum_{n=0}^{N-1} 4 \delta t \nu\left\|\nabla v^{n+1}\right\|_{L^{2}\left(\Omega_{t^{n+1}}\right)}^{2}+\sum_{n=0}^{N-1} 4 \delta t \tau^{n+1}\left\|\Pi_{h}^{\perp}\left(\left(\boldsymbol{a}-\boldsymbol{w}^{n+1}\right) \cdot \nabla v^{n+1}\right)\right\|_{L^{2}\left(\Omega_{t^{n+1}}\right)}^{2} \\
& +\int_{t^{n}}^{t^{n+1}} \int_{\Omega_{s}}(\nabla \cdot \boldsymbol{w}(s))\left(v^{n}\right)^{2} \mathrm{~d} \Omega \mathrm{d} s+\int_{t^{n}}^{t^{n+1}} \int_{\Omega_{s}}(\nabla \cdot \boldsymbol{w}(s))\left(2 v^{n}-v^{n-1}\right)^{2} \mathrm{~d} \Omega \mathrm{d} s .
\end{aligned}
$$


Manipulating the mesh velocity as for the BDF2-BDF ${ }_{\delta t}$ formulation (see Theorem 3.6) and applying the Gronwall Lemma we obtain the desired result.

Stability is now straightforward from Theorem 4.12 and Lemma 3.2:

COROLLARY 4.13 (Stability). There exists $\delta t_{\mathrm{cr}}^{2}$ such that, for $0<\delta t<\delta t_{\mathrm{cr}}^{2}$, the sequence $U_{h}$, solution of problem (2.17)-(2.22)-(2.14), is bounded as follows:

$$
\left\|U_{h}\right\|_{w}^{2} \leq C \sum_{n=0}^{N-1} \frac{\delta t}{\nu}\left\|f^{n+1}\right\|_{H^{-1}\left(\Omega_{t^{n+1}}\right)}^{2} .
$$

This stability result can be considered weak. However, we will see that this result is enough in order to obtain error estimates without constants depending on the Péclet number (except, of course, in the dependence of the continuous solution with this number), the original motivation of stabilization methods for convection-diffusion problems.

Let us obtain now error estimates for the BDF2-BDF2-OSS ${ }_{\delta t, h}$ formulation. We start with an auxiliary lemma that will be useful in the following:

LEMMA 4.14. Let $X=\left\{x^{n}\right\}_{n=0}^{N}$ and $V=\left\{v^{n}\right\}_{n=0}^{N}$ be two sequences of functions such that $x^{n}, v^{n} \in H^{p+1}\left(\Omega_{t^{n}}\right)$. Then, the bilinear form (2.22) satisfies the following bound:

$$
\begin{aligned}
B_{h}\left(X, \Pi_{h}^{\perp}(V)\right) \leq & C\left(\|X\|_{w}^{2}+\sum_{n=-1}^{N-1} \delta t\left(\tau^{n+1}\right)^{-1}\left\|\Pi_{h}^{\perp}\left(x^{n+1}\right)\right\|_{L^{2}\left(\Omega_{t^{n+1}}\right)}^{2}\right)^{\frac{1}{2}} \\
& \times\left(h^{2(p+1)} \sum_{n=-1}^{N-1} \delta t\left(\tau^{n+1}\right)^{-1}\left\|v^{n+1}\right\|_{H^{p+1}\left(\Omega_{t^{n+1}}\right)}^{2}\right)^{\frac{1}{2}} .
\end{aligned}
$$

Proof. From (2.22) we have that

$$
\begin{aligned}
B_{h}\left(X, \Pi_{h}^{\perp}(V)\right)= & \sum_{n=0}^{N-1} b_{h}\left(\boldsymbol{w}^{n+1} ; x^{n+1}, \Pi_{h}^{\perp}\left(v^{n+1}\right)\right)_{\Omega_{t^{n+1}}} \\
& +\sum_{n=1}^{N-1} \frac{1}{2 T}\left(3 x^{n+1}-4 x^{n}+x^{n-1}, \Pi_{h}^{\perp}\left(v^{n+1}\right)\right)_{\Omega_{t^{n+1}}} \\
& +\frac{1}{T}\left(x^{1}-x^{0}, \Pi_{h}^{\perp}\left(v^{1}\right)\right)_{\Omega_{t^{1}}}+\frac{1}{T}\left(x^{0}, \Pi_{h}^{\perp}\left(v^{0}\right)\right)_{\Omega_{0}},
\end{aligned}
$$

where

$$
\begin{aligned}
& \sum_{n=0}^{N-1} b_{h}\left(\boldsymbol{w}^{n+1} ; x^{n+1}, \Pi_{h}^{\perp}\left(v^{n+1}\right)\right)_{\Omega_{t^{n+1}}} \\
& =\sum_{n=0}^{N-1} \delta t\left[\nu\left(\nabla x^{n+1}, \nabla \Pi_{h}^{\perp}\left(v^{n+1}\right)\right)_{\Omega_{t^{n+1}}}+\left(\left(\boldsymbol{a}-\boldsymbol{w}^{n+1}\right) \cdot \nabla x^{n+1}, \Pi_{h}^{\perp}\left(v^{n+1}\right)\right)_{\Omega_{t^{n+1}}}\right. \\
& \left.\quad \quad+\tau^{n+1}\left(\Pi_{h}^{\perp}\left(\left(\boldsymbol{a}-\boldsymbol{w}^{n+1}\right) \cdot \nabla x^{n+1}\right),\left(\boldsymbol{a}-\boldsymbol{w}^{n+1}\right) \cdot \nabla \Pi_{h}^{\perp}\left(v^{n+1}\right)\right)_{\Omega_{t^{n+1}}}\right] .
\end{aligned}
$$

Now we have to bound every term of the right hand side in order to complete the proof. We 
start with the first term:

$$
\begin{aligned}
& \sum_{n=0}^{N-1} \delta t \nu\left(\nabla x^{n+1}, \nabla\left(\Pi_{h}^{\perp}\left(v^{n+1}\right)\right)\right)_{\Omega_{t^{n+1}}} \\
& \quad \leq\left(\sum_{n=0}^{N-1} \delta t \nu\left\|\nabla x^{n+1}\right\|_{L^{2}\left(\Omega_{t^{n+1}}\right)}^{2}\right)^{\frac{1}{2}}\left(\sum_{n=0}^{N-1} \delta t \nu\left\|\nabla \Pi_{h}^{\perp}\left(v^{n+1}\right)\right\|_{L^{2}\left(\Omega_{t^{n+1}}\right)}^{2}\right)^{\frac{1}{2}} .
\end{aligned}
$$

The second term in the right-hand side can be bounded as

$$
\begin{aligned}
& \sum_{n=0}^{N-1} \delta t\left(\left(\boldsymbol{a}-\boldsymbol{w}^{n+1}\right) \cdot \nabla x^{n+1}, \Pi_{h}^{\perp}\left(v^{n+1}\right)\right)_{\Omega_{t^{n+1}}} \\
& \leq\left(\sum_{n=0}^{N-1} \delta t \tau^{n+1}\left\|\Pi_{h}^{\perp}\left(\left(\boldsymbol{a}-\boldsymbol{w}^{n+1}\right) \cdot \nabla x^{n+1}\right)\right\|_{L^{2}\left(\Omega_{t^{n+1}}\right)}^{2}\right)^{\frac{1}{2}} \\
& \quad \times\left(\sum_{n=0}^{N-1} \delta t\left(\tau^{n+1}\right)^{-1}\left\|\Pi_{h}^{\perp}\left(v^{n+1}\right)\right\|_{L^{2}\left(\Omega_{t^{n+1}}\right)}^{2}\right)^{\frac{1}{2}}
\end{aligned}
$$

and the third term as

$$
\begin{aligned}
\sum_{n=0}^{N-1} \delta t \tau^{n+1} & \left(\Pi_{h}^{\perp}\left(\left(\boldsymbol{a}-\boldsymbol{w}^{n+1}\right) \cdot \nabla x^{n+1}\right),\left(\boldsymbol{a}-\boldsymbol{w}^{n+1}\right) \cdot \nabla \Pi_{h}^{\perp}\left(v^{n+1}\right)\right)_{\Omega_{t^{n+1}}} \\
\leq & \left(\sum_{n=0}^{N-1} \delta t \tau^{n+1}\left\|\Pi_{h}^{\perp}\left(\left(\boldsymbol{a}-\boldsymbol{w}^{n+1}\right) \cdot \nabla x^{n+1}\right)\right\|_{L^{2}\left(\Omega_{t^{n+1}}\right)}^{2}\right)^{\frac{1}{2}} \\
& \times\left(\sum_{n=0}^{N-1} \delta t \tau^{n+1}\left\|\Pi_{h}^{\perp}\left(\left(\boldsymbol{a}-\boldsymbol{w}^{n+1}\right) \cdot \nabla \Pi_{h}^{\perp}\left(v^{n+1}\right)\right)\right\|_{L^{2}\left(\Omega_{t^{n+1}}\right)}^{2}\right)^{\frac{1}{2}} .
\end{aligned}
$$

The term related to the time derivative is bounded after recalling assumption (2.21) for the stabilization parameter $\tau^{n+1}$ :

$$
\begin{aligned}
& \sum_{n=1}^{N-1} \frac{1}{T}\left(3 x^{n+1}-4 x^{n}+x^{n-1}, \Pi_{h}^{\perp}\left(v^{n+1}\right)\right)_{\Omega_{t^{n+1}}}+\frac{1}{T}\left(x^{1}-x^{0}, \Pi_{h}^{\perp}\left(v^{1}\right)\right)_{\Omega_{t^{1}}} \\
&+\frac{1}{T}\left(x^{0}, \Pi_{h}^{\perp}\left(v^{0}\right)\right)_{\Omega_{0}} \leq C\left(\sum_{n=-1}^{N-1} \delta t\left(\tau^{n+1}\right)^{-1}\left\|\Pi_{h}^{\perp}\left(x^{n+1}\right)\right\|_{L^{2}\left(\Omega_{t^{n+1}}\right)}\right)^{\frac{1}{2}} \\
& \times\left(\sum_{n=-1}^{N-1} \delta t\left(\tau^{n+1}\right)^{-1}\left\|\Pi_{h}^{\perp}\left(v^{n+1}\right)\right\|_{L^{2}\left(\Omega_{t^{n+1}}\right)}\right)^{\frac{1}{2}} .
\end{aligned}
$$

We now have to use (4.4) of Lemma 4.1 and the expression (2.20) of the stabilization parameter $\tau^{n+1}$ to conclude the proof. $\square$

To obtain the error estimate, we also need to invoke the coercivity of $B_{h}(\cdot, \cdot)$, which leads to

$$
B_{h}\left(E_{d}, E_{d}\right) \geq \beta_{2}\left\||| E_{d} \mid\right\|_{w}^{2}
$$


Subtracting the equation for the semi-discrete velocity and the discrete velocity we get

$$
\begin{aligned}
B_{h}\left(E_{d}, V_{h}\right) & =: \epsilon_{c}\left(V_{h}\right) \\
& =-\sum_{n=0}^{N-1} \delta t \tau^{n+1}\left(\Pi_{h}^{\perp}\left(\left(\boldsymbol{a}-\boldsymbol{w}^{n+1}\right) \cdot \nabla u^{n+1}\right),\left(\boldsymbol{a}-\boldsymbol{w}^{n+1}\right) \cdot \nabla v_{h}^{n+1}\right)_{\Omega_{t^{n+1}}} .
\end{aligned}
$$

Using the previous equation we can obtain

$$
B_{h}\left(E_{d}, E_{d}\right)=B_{h}\left(E_{d}, \Pi_{h}(U)-U\right)+\epsilon_{c}\left(U_{h}-\Pi_{h}(U)\right)
$$

The first term is due to the interpolation error, whereas the second one is the consistency error. In the following lemma we obtain a bound for the interpolation error:

LEMMA 4.15 (Interpolation error). The following inequality holds:

$$
\begin{aligned}
B_{h}\left(E_{d}, \Pi_{h}^{\perp}(U)\right) \leq & C\left(\left\|E_{d}\right\|\left\|_{w}^{2}+h^{2(p+1)} \sum_{n=-1}^{N-1} \delta t\left(\tau^{n+1}\right)^{-1}\right\| u^{n+1} \|_{H^{p+1}\left(\Omega_{t^{n+1}}\right)}^{2}\right)^{\frac{1}{2}} \\
& \times\left(h^{2(p+1)} \sum_{n=-1}^{N-1} \delta t\left(\tau^{n+1}\right)^{-1}\left\|u^{n+1}\right\|_{H^{p+1}\left(\Omega_{t^{n+1}}\right)}^{2}\right)^{\frac{1}{2}}
\end{aligned}
$$

Proof. Invoking lemma 4.14 and using the fact that $\Pi_{h}(U)-U=-\Pi_{h}^{\perp}(U)$ and $\Pi_{h}^{\perp}\left(E_{d}\right)=-\Pi_{h}^{\perp}(U)$, we immediately get the result. $\square$

In order to bound the consistency error we follow again the technique developed in Lemma 4.10. The only difference between these two cases is the term associated to the time derivative, which does not affect essentially the proof:

LEMMA 4.16 (Consistency error). The following inequality holds:

$$
\begin{aligned}
\epsilon_{c}\left(U_{h}-\Pi_{h}(U)\right) \leq & C\left(h^{2(p+1)} \sum_{n=0}^{N-1} \delta t\left(\tau^{n+1}\right)^{-1}\left\|u^{n+1}\right\|_{H^{p+1}\left(\Omega_{t^{n+1}}\right)}^{2}\right)^{\frac{1}{2}} \\
& \times\left(\left\|E_{d}\right\|_{w}^{2}+h^{2(p+1)} \sum_{n=0}^{N-1} \delta t\left(\tau^{n+1}\right)^{-1}\left\|u^{n+1}\right\|_{H^{p+1}\left(\Omega_{t^{n+1}}\right)}^{2}\right)^{\frac{1}{2}} .
\end{aligned}
$$

Again, we end with the desired convergence result, which is straight from Lemma 4.16 for the bound of the consistency error, Lemma 4.15 for the bound of the interpolation error and Theorem 4.12, that gives coercivity of the bilinear form:

THEOREM 4.17 (Convergence). There exist $\delta t_{\mathrm{cr}}^{2}$ such that, for $0<\delta t<\delta t_{\mathrm{cr}}^{2}$, the sequence of errors $E_{d}=U_{h}-U$ satisfies the following error estimate

$$
\left\|\left|E_{d}\left\|\left.\right|_{w} ^{2} \leq C h^{2(p+1)} \sum_{n=-1}^{N-1} \delta t\left(\tau^{n+1}\right)^{-1}\right\| u^{n+1} \|_{H^{p+1}\left(\Omega_{t^{n+1}}\right)}^{2} .\right.\right.
$$

This error estimate is optimal.

From this analysis, we can easily obtain stability and convergence results when the domain is fixed, that is, when the mesh velocity vanishes. 
4.3. Analysis of BDF2-OSS $\delta t, h$. The previous results are new even for fixed domains. The OSS stabilization method was analyzed in [11] using the backward Euler time integration. It can be easily seen that for fixed domains, i.e. when $\boldsymbol{w}^{n+1}=\mathbf{0}$, there is no critical time step size, the method becoming unconditionally stable. In this case, the problem to be solved reads as follows: find a sequence of finite element functions $U_{h}$ such that

$$
B_{h}\left(U_{h}, V_{h}\right)=L\left(V_{h}\right)
$$

with the bilinear form

$$
\begin{aligned}
B_{h}\left(U_{h}, V_{h}\right)= & \sum_{n=1}^{N-1}\left[\frac{1}{2 T}\left(3 u_{h}^{n+1}-4 u_{h}^{n}+u_{h}^{n-1}, v_{h}^{n+1}\right)+b_{h}\left(u_{h}^{n+1}, v_{h}^{n+1}\right)\right] \\
& +\frac{1}{T}\left(u_{h}^{1}-u_{h}^{0}, v_{h}^{1}\right)+b_{h}\left(u_{h}^{1}, v_{h}^{1}\right)+\frac{1}{T}\left(u_{h}^{0}, v_{h}^{0}\right),
\end{aligned}
$$

where now $b_{h}\left(u_{h}^{n+1}, v_{h}^{n+1}\right)$ denotes $b_{h}\left(\mathbf{0} ; u_{h}^{n+1}, v_{h}^{n+1}\right)$, with $b_{h}\left(\boldsymbol{w}^{n+1} ; u_{h}^{n+1}, v_{h}^{n+1}\right)$ defined in (2.19). The right-hand side linear form is given again by (2.14).

In this case two different sets of results are obtained. The first one with the weak norm $\||\cdot|\|_{w}$, and the second one with the strong norm $\||\cdot|\|_{s}$. The main difference is that in the second norm, $B_{h}(\cdot, \cdot)$, looses coercivity. This complicates the analysis.

We state the results with the norm $\left|\|\cdot \mid\|_{w}\right.$ in the following corollaries. Their proofs are straightforward from the previous analysis.

COROLLARY 4.18 (Stability). The sequence $U_{h}$ solution of problem (4.17) is bounded as follows:

$$
\left\|U_{h}\right\|\left\|_{w}^{2} \leq C \sum_{n=0}^{N-1} \frac{\delta t}{\nu}\right\| f^{n+1} \|_{H^{-1}(\Omega)}^{2} .
$$

for all $\delta t>0$.

Again, we denote by $U=\left\{u^{0}, u^{1}, u^{2}, \ldots, u^{N}\right\}$ the sequence of solutions of the second order semi-discrete problem (in time) (2.9)-(2.13)-(2.14), now with $\Omega_{t} \equiv \Omega$.

COROLlARY 4.19 (Convergence). The error sequence $E_{d}=U_{h}-U$ satisfies the following error estimate:

$$
\left\|\left|E_{d}\right|\right\|_{w}^{2} \leq C h^{2(p+1)} \sum_{n=-1}^{N-1} \delta t\left(\tau^{n+1}\right)^{-1}\left\|u^{n+1}\right\|_{H^{p+1}(\Omega)}^{2},
$$

for all $\delta t>0$.

The remaining of this section is devoted to improve these stability and convergence estimates. The improvement consists in obtaining estimates in the stronger norm $\left|\|\cdot \mid\| \|_{s}\right.$. This is possible for fixed domains, but we have not been able to obtain similar estimates as those presented next for moving domains. Nevertheless, some additional assumptions will be required. We will also remark the aspects that make the analysis of the BDF2-OSS $\mathrm{B}_{\delta t, h}$ method much more involved than that of the BDF1-OSS $\delta t, h$ formulation.

Let us introduce some new notation. We modify the bilinear form as follows:

$$
\begin{aligned}
B_{h}^{*}\left(U_{h}, V_{h}\right)= & \sum_{n=0}^{N-1} b_{h}\left(u_{h}^{n+1}, v_{h}^{n+1}\right)+\sum_{n=1}^{N-1} \frac{1}{2 T}\left(3 u_{h}^{n+1}-4 u_{h}^{n}+u_{h}^{n-1}, v_{h}^{n+1}\right) \\
& +\frac{1}{T}\left(u_{h}^{1}-u_{h}^{0}, v_{h}^{1}\right)+\frac{1}{T}\left(u_{h}^{0}, v_{h}^{0}\right)+\frac{1}{T \delta t}\left(u_{h}^{-1}, v_{h}^{-1}\right),
\end{aligned}
$$


and the right-hand-side linear form to:

$$
L^{*}\left(V_{h}\right)=\sum_{n=0}^{N-1} \delta t\left\langle f^{n+1}, v_{h}^{n+1}\right\rangle+\frac{1}{T}\left(u_{0}, v_{h}^{0}\right)+\frac{1}{T \delta t}\left(u_{1, h}-\Pi_{h}\left(u_{0}\right), v_{h}^{-1}\right),
$$

where $u_{0}$ is obviously the initial condition and $u_{1, h}$ is the solution at the first time step obtained with the scheme used to initialize the BDF2 scheme. For example, the BDF1 scheme can be used, and this is precisely what is assumed in the expression of $B_{h}^{*}(\cdot, \cdot)$. Note that now the sequences of finite element functions start at $n=-1$.

It is easily checked that the solution of the equation (2.9) with the bilinear form (2.12) is equivalent to

$$
B_{h}^{*}\left(U_{h}, V_{h}\right)=L^{*}\left(V_{h}\right) .
$$

Observe that this problem yields $u_{h}^{-1}=u_{1, h}-\Pi_{h}\left(u_{0}\right), u_{h}^{0}=\Pi_{h}\left(u_{0}\right)$ and $u_{h}^{1}=u_{1, h}$. The rest of the terms of the sequence of unknowns $U=\left\{u_{h}^{-1}, u_{h}^{0}, u_{h}^{2}, . ., u_{h}^{N}\right\}$ is the same as the solution of problem (4.17).

Let us introduce some additional ingredients. Given a sequence

$$
V=\left\{v^{-1}, v^{0}, v^{1}, v^{2}, \ldots, v^{N}\right\}
$$

we define

$$
\begin{aligned}
& d^{1, *}(V)=\left\{0,0,0, \delta v^{2}, \delta v^{3}, \ldots, \delta v^{N-1}, \delta v^{N}\right\} \\
& d^{2, *}(V)=\left\{0,0,-\delta v^{2},-\delta^{2} v^{2},-\delta^{2} v^{3}, \ldots,-\delta^{2} v^{N}, \delta v^{N}\right\}
\end{aligned}
$$

These operators on sequences have the following property: for all sequences $X=\left\{x^{n}\right\}_{n=-1}^{N}$ it holds that

$$
\begin{aligned}
B_{h}^{*}\left(X, d^{2, *}(V)\right)= & \sum_{n=1}^{N-1} b_{h}\left(\delta x^{n+1}, \delta v^{n+1}\right)+\sum_{n=2}^{N-1} \frac{1}{2 T}\left(3 \delta x^{n+1}-4 \delta x^{n}+\delta x^{n-1}, \delta v^{n+1}\right) \\
& +\frac{3}{2 T}\left(\delta x^{2}-\delta x^{1}, \delta v^{2}\right) \\
= & B_{h}^{*}\left(d^{1, *}(X), d^{1, *}(V)\right)+\frac{1}{2 T}\left(\delta x^{1}, \delta v^{3}-3 \delta v^{2}\right) .
\end{aligned}
$$

REMARK 4.3. The previous property is not satisfied for moving domains due to the fact that the convective velocity changes at every time step. It introduces an extra term $b_{h}\left(\delta \boldsymbol{w}^{n+1} ; u^{n}, \delta v^{n+1}\right)_{\Omega_{t^{n+1}}}$ that can not be bounded as required in the following analysis.

In the next theorem we obtain $\Lambda$-coercivity for the norm ||$|\cdot| \|_{s}$.

THEOREM 4.20 ( $\Lambda$-coercivity). Let $V=\left\{v^{n}\right\}_{n=-1}^{N}$ be a sequence of functions such that $v^{n} \in \mathcal{V}(\Omega), n=0,1, \ldots, N$, and $v^{-1}=v^{1}-v^{0}$, and consider the operator

$$
\Lambda(V)=V+\left\{0,0, \frac{1}{4}\left\{\tau^{n+1} \Pi_{h}\left(\boldsymbol{a} \cdot \nabla v^{n+1}\right)\right\}_{0}^{N-1}\right\}+\delta t^{-1} d^{2, *}(V) .
$$

Then, the bilinear form $B_{h}^{*}(\cdot, \cdot)$ is $\Lambda$-coercive. In particular, the following inequality holds:

$$
B_{h}^{*}(V, \Lambda(V)) \geq \beta_{2}\left(\left.\|V\|\right|_{s} ^{2}+\delta t^{-1}\left\|\mid d^{1, *}(V)\right\|_{w}^{2}+\frac{1}{T \delta t}\left\|v^{-1}\right\|_{L^{2}(\Omega)}^{2}\right)
$$


for a certain constant $\beta_{2}>0$.

Proof. It can be easily shown that

$$
\begin{aligned}
B_{h}^{*}(V, 4 V)= & \sum_{n=0}^{N-1} 4 b_{h}\left(v^{n+1}, v^{n+1}\right)+\sum_{n=2}^{N-1} \frac{4}{2 T}\left(3 v^{n+1}-4 v^{n}+v^{n-1}, v^{n+1}\right) \\
& +\frac{4}{T}\left(v^{1}-v^{0}, v^{1}\right)+\frac{4}{T}\left(v^{0}, v^{0}\right)+\frac{4}{T \delta t}\left(v^{-1}, v^{-1}\right) \\
\geq & \sum_{n=0}^{N-1} 4\left[\delta t \nu\left\|\nabla v^{n+1}\right\|_{L^{2}(\Omega)}^{2}+\delta t \tau^{n+1}\left\|\Pi_{h}^{\perp}\left(\boldsymbol{a} \cdot \nabla v^{n+1}\right)\right\|_{L^{2}(\Omega)}^{2}\right] \\
+ & \frac{1}{T}\left[\left\|v^{N+1}\right\|_{L^{2}(\Omega)}^{2}+\sum_{n=1}^{N-1}\left\|\delta^{2} v^{n+1}\right\|_{L^{2}(\Omega)}^{2}+2\left\|v^{0}\right\|_{L^{2}(\Omega)}^{2}+\frac{4}{\delta t}\left\|v^{-1}\right\|_{L^{2}(\Omega)}^{2}\right] .
\end{aligned}
$$

In order to obtain stability for the component of the convective term in the finite element space, we use as test function the sequence $\left\{0,0,\left\{\tau^{n+1} \Pi_{h}\left(\boldsymbol{a} \cdot \nabla v^{n+1}\right)\right\}_{0}^{N-1}\right\}=: \Pi_{0}(\tau, V)$, that is starting with 0 in the component -1 and 0 . Exactly as in the proof of Theorem 4.5, we now obtain:

$$
\begin{gathered}
B_{h}^{*}\left(V, \Pi_{0}(\tau, V)\right) \geq \sum_{n=0}^{N-1} \phi^{n+1} \delta t \tau^{n+1}\left\|\Pi_{h}\left(\boldsymbol{a} \cdot \nabla v^{n+1}\right)\right\|_{L^{2}(\Omega)}^{2} \\
-\sum_{n=0}^{N-1}\left[\delta t \nu\left\|\nabla v^{n+1}\right\|_{L^{2}(\Omega)}^{2}+\delta t \tau^{n+1}\left\|\Pi_{h}^{\perp}\left(\boldsymbol{a} \cdot \nabla v^{n+1}\right)\right\|_{L^{2}(\Omega)}^{2}\right] \\
-\sum_{n=1}^{N-1} \frac{1}{4 T}\left\|3 v^{n+1}-4 v^{n}+v^{n-1}\right\|_{L^{2}(\Omega)}^{2}-\frac{1}{T}\left\|v^{1}-v^{0}\right\|_{L^{2}(\Omega)}^{2},
\end{gathered}
$$

with the expression of $\phi^{n+1}$ given in (4.8). We do not have control over the term related to the time derivative, needing a further step. We use now as test function $d^{2, *}(V)$. From the first step in (4.21) it follows that

$$
\begin{aligned}
\delta t^{-1} B_{h}^{*}\left(V, 4 d^{2, *}(V)\right) & \geq \delta t^{-1} \mid\left\|d^{1, *}(V)\right\|_{w}^{2}-\frac{3}{T \delta t}\left\|\delta v^{1}\right\|_{L^{2}(\Omega)}^{2} \\
& =\delta t^{-1}\left\|\left|d^{1, *}(V)\left\|\left.\right|_{w} ^{2}-\frac{3}{T \delta t}\right\| v^{-1} \|_{L^{2}(\Omega)}^{2} .\right.\right.
\end{aligned}
$$

Combining the previous inequalities and invoking the Gronwall lemma (without any assumption over the time step size) we can conclude the proof of the theorem.

REMARK 4.4. In equation (4.22) we do not have control over the term associated to the time derivative. It makes the analysis for the second order method more intricate than for the first order one, for which the time derivative term is easily controlled (see (4.7)). The control of this term has motivated the introduction of $d^{2, *}(V)$ in the test sequence used.

In order to obtain stability it remains to prove some kind of continuity with respect to the operator $\Lambda$. This is what the next theorem states: 
THEOREM 4.21 ( $\Lambda$-continuity). The following inequality holds:

$$
\begin{aligned}
& L^{*}(\Lambda(V)) \\
& \leq\left(\sum_{n=0}^{N-1} \frac{\delta t}{\nu}\left\|f^{n+1}\right\|_{H^{-1}(\Omega)}^{2}+\sum_{n=1}^{N-1} \frac{\delta t^{2}}{\nu}\left\|D_{1} f^{n+1}\right\|_{H^{-1}(\Omega)}^{2}\right. \\
& \left.\quad+\frac{1}{T}\left\|u_{0}\right\|_{L^{2}(\Omega)}^{2}+\frac{\delta t}{T}\left\|\frac{u_{1, h}-\Pi_{h}\left(u_{0}\right)}{\delta t}\right\|_{L^{2}(\Omega)}^{2}\right)^{\frac{1}{2}} \\
& \quad \times\left(\|V\|_{s}^{2}+\delta t^{-1}\left\|d^{1, *}(V)\right\|\left\|_{w}^{2}+\frac{1}{T}\right\| v^{0}\left\|_{L^{2}(\Omega)}^{2}+\frac{1}{T \delta t}\right\| v^{-1} \|_{L^{2}(\Omega)}^{2}\right)^{\frac{1}{2}} .
\end{aligned}
$$

Proof. The following inequalities can be easily obtained:

$$
\begin{gathered}
L^{*}(V) \leq\left(\sum_{n=0}^{N-1} \frac{\delta t}{\nu}\left\|f^{n+1}\right\|_{H^{-1}(\Omega)}^{2}+\frac{1}{T}\left\|u_{0}\right\|_{L^{2}(\Omega)}^{2}+\frac{\delta t}{T}\left\|\frac{u_{1, h}-\Pi_{h}\left(u_{0}\right)}{\delta t}\right\|_{L^{2}(\Omega)}^{2}\right)^{\frac{1}{2}} \\
\times\left(\sum_{n=0}^{N-1} \delta t \nu\left\|\nabla v^{n+1}\right\|_{L^{2}(\Omega)}^{2}+\frac{1}{T}\left\|v^{0}\right\|_{L^{2}(\Omega)}^{2}+\frac{1}{T \delta t}\left\|v^{-1}\right\|_{L^{2}(\Omega)}^{2}\right)^{\frac{1}{2}}, \\
L^{*}\left(\delta t^{-1} d^{2, *}(V)\right) \leq\left(\sum_{n=1}^{N-1} \frac{\delta t^{2}}{\nu}\left\|D_{1} f^{n+1}\right\|_{H^{-1}(\Omega)}^{2}\right)^{\frac{1}{2}}\left(\sum_{n=1}^{N-1} \delta t^{2} \nu\left\|D_{1} v^{n+1}\right\|_{H^{1}(\Omega)}^{2}\right)^{\frac{1}{2}}, \\
L^{*}\left(\Pi_{0}(\tau, V)\right) \leq\left(\sum_{n=0}^{N-1} \frac{\delta t}{\nu}\left\|f^{n+1}\right\|_{H^{-1}(\Omega)}^{2}\right)^{\frac{1}{2}} \\
\times\left(\sum_{n=0}^{N-1} \delta t \nu\left\|\tau^{n+1} \nabla\left(\Pi_{h}\left(\boldsymbol{a} \cdot \nabla v^{n+1}\right)\right)\right\|_{L^{2}(\Omega)}^{2}\right)^{\frac{1}{2}},
\end{gathered}
$$

and

$$
\begin{aligned}
\nu\left\|\tau^{n+1} \nabla\left(\Pi_{h}\left(\boldsymbol{a} \cdot \nabla v^{n+1}\right)\right)\right\|_{L^{2}(\Omega)}^{2} & \leq \frac{C_{\mathrm{inv} \nu}^{2} \nu}{h^{2}}\left(\tau^{n+1}\right)^{2}\left\|\Pi_{h}\left(\boldsymbol{a} \cdot \nabla v^{n+1}\right)\right\|_{L^{2}(\Omega)}^{2} \\
& \leq C \tau^{n+1}\left\|\boldsymbol{a} \cdot \nabla v^{n+1}\right\|_{L^{2}(\Omega)}^{2} .
\end{aligned}
$$

From all these inequalities the theorem follows easily. $\square$

The two previous theorems lead to the following stability result:

COROLLARY 4.22 (Stability II). The sequence $U_{h}$, solution of problem (4.17), is bounded as follows:

$$
\begin{aligned}
\left.\left\|U_{h}\right\|\right|_{s} ^{2}+ & \delta t^{-1}\left\|\left|d^{1, *} U_{h} \|\right|_{w}^{2}\right. \\
\leq & C\left(\sum_{n=0}^{N-1} \frac{\delta t}{\nu}\left\|f^{n+1}\right\|_{H^{-1}(\Omega)}^{2}+\sum_{n=1}^{N-1} \frac{\delta t^{2}}{\nu}\left\|D_{1} f^{n+1}\right\|_{H^{-1}(\Omega)}^{2}\right. \\
& \left.+\frac{1}{T}\left\|u^{0}\right\|_{L^{2}(\Omega)}^{2}+\frac{\delta t}{T}\left\|\frac{u_{1, h}-\Pi_{h}\left(u_{0}\right)}{\delta t}\right\|_{L^{2}(\Omega)}^{2}\right)
\end{aligned}
$$


for all $\delta t>0$.

Obviously, this stability bounds makes sense if the initialization is such that the last term on the right-hand-side is bounded. Using for example the backward Euler scheme, this poses a mild condition on $\delta t$ and $h$. In particular, if $h^{p+1} \leq C \delta t$, it is easy to show that this last term is bounded.

The final result we obtain is an error estimate in the strong norm $\left|\|\cdot \mid\|_{s}\right.$. At this point we introduce the sequence $U=\left\{u_{h}^{-1}, u^{0}, u^{1}, u^{2}, \ldots, u^{N}\right\}$, that consists of the sequence of solutions of the semi-discrete problem (2.9)-(2.11) supplemented with $u_{h}^{-1}$ at $n=-1$. It can be easily checked that this sequence satisfies

$$
B_{h}^{*}(U, V)=L^{*}(V)-\epsilon_{c}(V) .
$$

Thus, $E_{d}:=U_{h}-U=\left\{0, u_{h}^{0}-u^{0}, u_{h}^{1}-u^{1}, \ldots, u_{h}^{N}-u^{N}\right\}$ satisfies

$$
B_{h}^{*}\left(E_{d}, V_{h}\right)=\epsilon_{c}\left(V_{h}\right) .
$$

We point out that for fixed domains the critical time step size does not appear anymore due to the fact that $\boldsymbol{w}=\mathbf{0}$. The method is unconditionally stable, as expected.

We stress the fact that $e_{d}^{-1} \neq e_{d}^{1}-e_{d}^{0}$, and therefore $E_{d}$ does not verify the statement of Theorem 4.20. The only place where the fact that $v^{-1}=v^{1}-v^{0}$ is used is in (4.23). When the test sequence does not satisfy the assumption $v^{-1}=v^{1}-v^{0}$ of Theorem 4.20, we have to modify the $\Lambda$-coercivity proved in this theorem as follows:

$$
\frac{4}{T \delta t}\left\|\delta e_{d}^{1}\right\|_{L^{2}(\Omega)}^{2}+B_{h}^{*}\left(E_{d}, \Lambda\left(E_{d}\right)\right) \geq \beta_{2}\left(\left\||| E_{d}\right\|_{s}^{2}+\delta t^{-1} \mid\left\|d^{1, *}\left(E_{d}\right)\right\| \|_{w}^{2}\right) .
$$

With the expression of $\Lambda(\cdot)$ given in Theorem 4.20 we arrive to

$$
\begin{aligned}
B_{h}^{*}\left(E_{d}, \Lambda\left(E_{d}\right)\right)= & B_{h}^{*}\left(E_{d}, E_{d}\right)+\frac{1}{4} \epsilon_{c}\left(\Pi_{0}\left(\tau, E_{d}\right)\right)+\delta t^{-1} B_{h}^{*}\left(E_{d}, d^{2, *} E_{d}\right) \\
= & B_{h}^{*}\left(E_{d}, \Pi_{h}(U)-U\right)+\epsilon_{c}\left(U_{h}-\Pi_{h}(U)\right)+\frac{1}{4} \epsilon_{c}\left(\Pi_{0}\left(\tau, E_{d}\right)\right) \\
& +\delta t^{-1} B_{h}^{*}\left(E_{d}, d^{2, *}\left(\Pi_{h}(U)-U\right)\right)+\delta t^{-1} \epsilon_{c}\left(d^{2, *}\left(U_{h}-\Pi_{h}(U)\right)\right) .
\end{aligned}
$$

Again, we group the different terms as interpolation and consistency errors and bound them separately in the next lemmas.

LEMMA 4.23 (Interpolation error). The following inequality holds:

$$
\begin{aligned}
& B_{h}\left(E_{d}, \Pi_{h}^{\perp}(U)\right)+\delta t^{-1} B_{h}^{*}\left(E_{d}, d^{2, *}\left(\Pi_{h}^{\perp}(U)\right)\right. \\
& \leq\left(\left\|E_{d}\right\|\left\|_{w}^{2}+\delta t^{-1}\right\| d^{1, *}\left(E_{d}\right) \|\left.\right|_{w} ^{2}\right. \\
& \left.\quad+h^{2(p+1)} \sum_{n=0}^{N-1} \delta t\left(\tau^{n+1}\right)^{-1}\left(\left\|u^{n+1}\right\|_{H^{p+1}(\Omega)}^{2}+\left\|\sqrt{\delta t} D_{1} u^{n+1}\right\|_{H^{p+1}(\Omega)}^{2}\right)\right)^{\frac{1}{2}} \\
& \quad \times\left(h^{2(p+1)} \sum_{n=0}^{N-1} \delta t\left(\tau^{n+1}\right)^{-1}\left(\left\|u^{n+1}\right\|_{H^{p+1}(\Omega)}^{2}+\left\|\sqrt{\delta t} D_{1} u^{n+1}\right\|_{H^{p+1}(\Omega)}^{2}\right)\right)^{\frac{1}{2}} .
\end{aligned}
$$


Proof. The bound for the first term of the left-hand side of the inequality is easily obtained from the proof of Lemma 4.15, since $e_{d}^{-1}=0$. For the second term we use property (4.21) and again the fact that $e_{d}^{-1}=0$, getting

$$
\begin{aligned}
& B_{h}^{*}\left(E_{d}, d^{2, *}\left(\Pi_{h}^{\perp}(U)\right)\right) \\
& =B_{h}\left(d^{1, *}\left(E_{d}\right), d^{1, *}\left(\Pi_{h}^{\perp}(U)\right)\right)-\frac{1}{2 T}\left(\delta e_{d}^{1}, \delta\left(\Pi_{h}\left(u^{3}\right)-3 \Pi_{h}\left(u^{2}\right)\right)\right) .
\end{aligned}
$$

Note that when we write $B_{h}\left(d^{1, *}\left(E_{d}\right), d^{1, *}\left(\Pi_{h}^{\perp}(U)\right)\right)$ we eliminate the element -1 of the sequences to apply the bilinear form $B_{h}(\cdot, \cdot)$.

Using Lemma 4.14 we get

$$
\begin{aligned}
& \delta t^{-1} B_{h}\left(d^{1, *}\left(E_{d}\right), d^{1, *}\left(\Pi_{h}^{\perp}(U)\right)\right) \\
& \leq C\left(\delta t ^ { - 1 } \left\|\left|d^{1, *} E_{d}\left\|\left.\right|_{w} ^{2}+h^{2(p+1)} \sum_{n=1}^{N-1} \delta t\left(\tau^{n+1}\right)^{-1}\right\| \sqrt{\delta t} D_{1} u^{n+1} \|_{H^{p+1}(\Omega)}^{2}\right)^{\frac{1}{2}}\right.\right. \\
& \quad \times\left(h^{2(p+1)} \sum_{n=1}^{N-1} \delta t\left(\tau^{n+1}\right)^{-1}\left\|\sqrt{\delta t} D_{1} u^{n+1}\right\|_{H^{p+1}(\Omega)}^{2} \cdot\right.
\end{aligned}
$$

Exploiting the fact that $\Pi_{h}^{\perp}\left(e_{d}^{1}\right)=\Pi_{h}\left(u^{1}\right)-u^{1}$ we can easily get that

$$
\begin{aligned}
& \frac{1}{2 T \delta t}\left(\delta e_{d}^{1}, \delta\left(\Pi_{h}\left(u^{3}\right)-3 \Pi_{h}\left(u^{2}\right)\right)\right) \\
& \leq C h^{2(p+1)} \sum_{n=0}^{2} \delta t\left(\tau^{n+1}\right)^{-1}\left\|\sqrt{\delta t} D_{1} u^{n+1}\right\|_{H^{p+1}(\Omega)}^{2}
\end{aligned}
$$

The proof is concluded. $\square$

LEMMA 4.24 (Consistency error). The following inequality holds:

$$
\begin{aligned}
& \epsilon_{c}\left(U_{h}-\Pi_{h}(U)+\frac{1}{4} \Pi_{0}\left(\tau, E_{d}\right)+\delta t^{-1} d^{2, *}\left(U_{h}-\Pi_{h}(U)\right)\right) \\
& \leq C\left(h^{2(p+1)} \sum_{n=0}^{N-1} \delta t\left(\tau^{n+1}\right)^{-1}\left(\left\|u^{n+1}\right\|_{H^{p+1}(\Omega)}^{2}+\left\|\sqrt{\delta t} D_{1} u^{n+1}\right\|_{H^{p+1}(\Omega)}^{2}\right)\right)^{\frac{1}{2}} \times \\
& \left(\left\||| E_{d}\right\|_{w}^{2}+h^{2(p+1)} \sum_{n=0}^{N-1} \delta t\left(\tau^{n+1}\right)^{-1}\left(\left\|u^{n+1}\right\|_{H^{p+1}(\Omega)}^{2}+\left\|\sqrt{\delta t} D_{1} u^{n+1}\right\|_{H^{p+1}(\Omega)}^{2}\right)\right)^{\frac{1}{2}} .
\end{aligned}
$$

Proof. Due to the fact that $e_{d}^{-1}=0$ we can take profit from the bounds obtained in 
Lemmas 4.10 and 4.16. The remaining term associated to $d^{2, *}(\cdot)$ can be bounded as follows:

$$
\begin{aligned}
\epsilon_{c}\left(\delta t^{-1} d^{2, *}\left(U_{h}-\Pi_{h}(U)\right)\right)= & \sum_{n=1}^{N-1} \tau^{n+1}\left(\Pi_{h}^{\perp}\left(\boldsymbol{a} \cdot \nabla \delta u^{n+1}\right), \boldsymbol{a} \cdot \nabla \Pi_{h}^{\perp}\left(\delta u^{n+1}\right)\right) \\
= & \sum_{n=1}^{N-1} \tau^{n+1}\left(\Pi_{h}^{\perp}\left(\lambda\left(\delta u^{n+1}\right)\right), \boldsymbol{a} \cdot \nabla \Pi_{h}^{\perp}\left(\delta u^{n+1}\right)\right) \\
\leq & C\left(\sum_{n=1}^{N-1} \delta t \tau^{n+1}\left\|\Pi_{h}^{\perp}\left(\sqrt{\delta t} \lambda\left(D_{1} u^{n+1}\right)\right)\right\|_{L^{2}(\Omega)}^{2}\right)^{\frac{1}{2}} \\
& \quad \times\left(h^{2(p+1)} \sum_{n=1}^{N-1} \delta t\left(\tau^{n+1}\right)^{-1}\left\|\sqrt{\delta t} D_{1} u^{n+1}\right\|_{H^{p+1}(\Omega)}^{2}\right)^{\frac{1}{2}},
\end{aligned}
$$

with $\lambda(\cdot)$ introduced in Lemma 4.10. The term related to $\lambda\left(D_{1} u^{n+1}\right)$ can be easily bounded from the expression of $\tau^{n+1}$, assumption (2.19) and the interpolation error estimate (4.3), as pointed out in Lemma 4.10.

We end with the convergence result of the method in the norm ||$|\cdot| \|_{s}$ :

THEOREM 4.25 (Convergence II). The sequence of errors $E_{d}=U_{h}-U$ satisfies the following error estimate:

$$
\begin{aligned}
\left\|E_{d} \mid\right\|_{s}^{2} \leq & C h^{2(p+1)}\left[\sum_{n=0}^{N-1} \delta t\left(\tau^{n+1}\right)^{-1}\left(\left\|u^{n+1}\right\|_{H^{p+1}(\Omega)}^{2}+\left\|\sqrt{\delta t} D_{1} u^{n+1}\right\|_{H^{p+1}(\Omega)}^{2}\right)\right. \\
& \left.+\left(\tau^{1}\right)^{-1}\left\|u^{1}\right\|_{H^{p+1}(\Omega)}+\left(\tau^{1}\right)^{-1}\left\|u^{0}\right\|_{H^{p+1}(\Omega)}\right]
\end{aligned}
$$

for all $\delta t>0$.

Proof. Using Lemma 4.23 and Lemma 4.24 in expressions (4.24) and (4.25), we can easily get the desired bound for $\left\|\left|E_{d}\right|\right\|_{s}^{2}$ in terms of $\frac{4}{T \delta t}\left\|\delta e_{d}^{1}\right\|_{L^{2}(\Omega)}^{2}$. Using as initialization the backward Euler scheme and the convergence result of Theorem 4.11 for the semidiscrete problem, it follows that

$$
\begin{aligned}
\frac{1}{T \delta t}\left\|\delta e_{d}^{1}\right\|_{L^{2}(\Omega)}^{2} & \leq \frac{C}{T \delta t}\left(\left\|e_{d}^{1}\right\|_{L^{2}(\Omega)}^{2}+\left\|u^{0}-\Pi_{h}\left(u^{0}\right)\right\|_{L^{2}(\Omega)}^{2}\right) \\
& \leq C\left(\tau^{1}\right)^{-1} h^{2(p+1)}\left(\left\|u^{1}\right\|_{H^{p+1}(\Omega)}+\left\|u^{0}\right\|_{H^{p+1}(\Omega)}\right)
\end{aligned}
$$

from which we obtain the desired result. $\square$

REMARK 4.5. From (4.26) it is seen that we need $\left\{\sqrt{\delta t} D_{1} u^{n+1}\right\}$ bounded in the norm of $\ell^{2}\left(H^{p+1}(\Omega)\right)$. This can be understood as additional regularity on the data or as an additional assumption on the asymptotic behavior of the time step size in terms of $h$. From the semidiscrete equation, it is immediate to bound $\left\|D_{1} u^{n+1}\right\|_{H^{q}(\Omega)}$ in terms of the $H^{q}(\Omega)$-norm of the rest of the terms of the equation. In particular, the viscous term implies that the $H^{q}(\Omega)$-norm of $D_{1} u^{n+1}$ can be bounded in terms of the $H^{q+2}(\Omega)$-norm of $u^{n+1}$. If only the $H^{p+1}(\Omega)$ norm of $u^{n+1}$ is bounded, we have to take $q=p-1$, and thus $h^{2(p+1)}\left\|\sqrt{\delta t} D_{1} u^{n+1}\right\|_{H^{p+1}(\Omega)}^{2}$ has to be replaced by $h^{2(p-1)}\left\|\sqrt{\delta t} D_{1} u^{n+1}\right\|_{H^{p-1}(\Omega)}^{2}$, and therefore we need $\delta t \leq C h^{4}$ in order to maintain the optimal order of accuracy. 
5. Conclusions. In this paper we have analyzed a stabilized finite element method to approximate the convection-diffusion equation on moving domains. The Orthogonal Subgrid Scale formulation has been used as stabilization technique and an ALE framework has been used in order to deal with moving domains.

In the first part of the paper we have analyzed the semi-discrete problem (in time). Two methods have been considered. A first order accurate method, where the time derivatives are computed using the BDF1 scheme, and a second order accurate method, where the BDF2 scheme has been used. In this analysis it is easy to identify the error introduced by the ALE formulation. The mesh velocity is computed as the time derivative of the mesh displacement. The numerical approximation of this time derivative is the only source of error introduced by the ALE formulation. As a conclusion, in order to keep the accuracy of a $k$-th order (in time) method on fixed domains, we must compute the mesh velocity using a time integration scheme of, at least, order $k$ of accuracy. The only negative aspect is that unconditional stable methods for fixed domains become conditionally stable.

In the second part of the paper we have analyzed a stabilized transient convectiondiffusion equation in an ALE framework. We have introduced the concept of $\Lambda$-coercivity that has been used for obtaining stability results and error estimates. It has been shown that the OSS method can be easily extended to transient problems. For the BDF1 time integration scheme we have stability of the convective term norm, as usual when using stabilization techniques. The analysis of BDF2 is more complicated. We only have control over the orthogonal projection of the convective term. However, optimal convergence results with constants that do not depend on the Péclet number can be proved. Finally, for fixed domains, we have been able to recover stronger stability and convergence involving the full norm of the convective term, but the analysis is much more involved and requires more regularity assumptions.

\section{REFERENCES}

[1] S. BADIA AND R. CODINA, On some fluid-structure iterative algorithms using pressure segregation methods. Application to aeroelasticity, International Journal for Numerical Methods in Engineering, (submitted).

[2] D. Boffi And L. Gastaldi, Stability and geometric conservation laws for ALE formulation, Computer Methods in Applied Mechanics and Engineering, 193 (2004), pp. 4717-4739.

[3] M. BRAACK AND E. BURMAN, Local projection stabilization for the Oseen problem and its interpretation as a variational multiscale method, SIAM Journal on Numerical Analysis, (to appear).

[4] S.C. BRENNER AND L.R. SCOTT, The mathematical theory of finite element methods, Springer-Verlag, 1994.

[5] A.N. BROOKS AND T.J.R. HugheS, Streamline upwind / Petrov-Galerkin formulations for convection dominated flows with particular emphasis on the incompressible Navier-Stokes equation, Computer Methods in Applied Mechanics and Engineering, 32 (1982), pp. 199-259.

[6] R. CoDINA, Comparison of some finite element methods for solving the diffusion-convection-reaction equation, Computer Methods in Applied Mechanics and Engineering, 156 (1998), pp. 185-210.

[7] - Stabilization of incompressibility and convection through orthogonal sub-scales in finite element methods, Computer Methods in Applied Mechanics and Engineering, 190 (2000), pp. 1579-1599.

[8] - Pressure stability in fractional step finite element methods for incompressible flows, Journal of Computational Physics, 170 (2001), pp. 112-140.

[9] - A stabilized finite element method for generalized stationary incompressible flows, Computer Methods in Applied Mechanics and Engineering, 190 (2001), pp. 2681-2706.

[10] R. CODINA AND S. BADIA, On some pressure segregation methods of fractional-step type for the finite element approximation of incompressible flow problems, Computer Methods in Applied Mechanics and Engineering, (in press).

[11] R. CODINA AND J. BLASCO, Analysis of a stabilized finite element approximation of the transient convectiondiffusion-reaction equation using orthogonal subscales, Computing and Visualization in Science, 4 (2002), pp. 167-174.

[12] J. Donea, P. Fasoli-Stella, And S. Giuliani, Lagrangian and Eulerian finite element techniques for transient fluid structure interaction problems, in Transactions Fourth SMIRT, 1977, p. B1/2.

[13] C. FARHAT, P. GeuZAine, And C. GRAndmont, The discrete geometric conservation law and the non- 
linear stability of ALE schemes for the solution of flow problems on moving grids, Journal of Computational Physics, 174 (2001), pp. 669-692.

[14] L. FORMAGGIA AND F. NOBILE, A stability analysis for the Arbitrary Lagrangian Eulerian formulation with finite elements, East-West J. Num. Math., 7 (1999), pp. 105-132.

[15] Stability analysis of second-order time accurate schemes for ALE-FEM, Computer Methods in Applied Mechanics and Engineering, 193 (2004), pp. 4097-4116.

[16] P. Geuzaine, C. Grandmont, And C. FARHAT, Design and analysis of ALE schemes with provable second-order time accuracy for inviscid and viscous flow simulations, Journal of Computational Physics, 191(1) (2003), pp. 206-227.

[17] V. GiRAUlt AND P.A. RaVIART, Finite element methods for Navier-Stokes equations, Springer-Verlag, 1986.

[18] J.L. GUERMOND, Subgrid stabilization of Galerkin approximations of linear contraction semi-groups of class $\mathcal{C}^{0}$, Computing and Visualization in Science, 2 (1999), pp. 131-138.

[19] J.G. HEYWOOD AND R. RANNACHER, Finite element approximation of the nonstationary Navier-Stokes problem. IV: Error analysis for second-order time discretization, SIAM Journal on Numerical Analysis, 27 (1990), pp. 353-384.

[20] T.J.R. HugheS, Multiscale phenomena: Green's function, the Dirichlet-to-Neumann formulation, subgrid scale models, bubbles and the origins of stabilized formulations, Computer Methods in Applied Mechanics and Engineering, 127 (1995), pp. 387-401.

[21] T.J.R. HugheS, G.R. FeiJóo, L. MAZZEI, AND J.B. QuinCY, The variational multiscale method-a paradigm for computational mechanics, Computer Methods in Applied Mechanics and Engineering, 166 (1998), pp. 3-24.

[22] T.J.R. Hughes, L.P. FranCA, AND G.M. Hulbert, A new finite element formulation for computational fluid dynamics: VIII. The Galerkin / least-squares method for advective-diffusive equations, Computer Methods in Applied Mechanics and Engineering, 73 (1989), pp. 173-189.

[23] F. NobILE, Numerical Approximation of Fluid-Structure Interaction problems with application to Haemodynamics, PhD thesis, École Polytechnique Fédérale de Lausanne, 2001. 